Supporting Information

for

\title{
Synthesis of the Azaspiracid-1 Trioxadispiroketal
}

Lisa K. Geisler, Son Nguyen, and Craig J. Forsyth*

Department of Chemistry
University of Minnesota
Minneapolis, Minnesota 55455

Contents: Experimentals and characterization data for key compounds, annotated ROESY plot of AZA-1, and copies of proton and carbon NMR spectra. 
General: All air and moisture sensitive reactions were carried out under argon in oven-dried glassware using standard syringe, cannula, and septa techniques. Tetrahydrofuran and diethyl ether were distilled from $\mathrm{Na}$ /benzophenone ketyl under nitrogen. Methylene chloride, triethylamine and toluene were distilled from $\mathrm{CaH}_{2}$ under nitrogen. Copper iodide was dried under high vacuum for 24 hours with heating. Flash column chromatography was performed using Baker Flash silica gel $60(40 \mu \mathrm{m})$. NMR spectra were obtained in $\mathrm{CDCl}_{3}$ and referenced to residual $\mathrm{CHCl}_{3}$ at $7.27 \mathrm{ppm}\left({ }^{1} \mathrm{H}\right)$ and $77.0 \mathrm{ppm}\left({ }^{13} \mathrm{C}\right)$. Optical rotations were obtained using a JASCO DIP-370 digital polarimeter at the sodium D line $(589 \mathrm{~nm})$ and were reported in concentration $(c=\mathrm{g} / 100 \mathrm{~mL})$ at $23{ }^{\circ} \mathrm{C}$. Infrared (IR) spectra were obtained using a MIDAC Prospect FT-IR spectrophotometer. High and low resolution mass spectral analysis (EI) was performed on a Bruker Biotof II mass spectrometer.

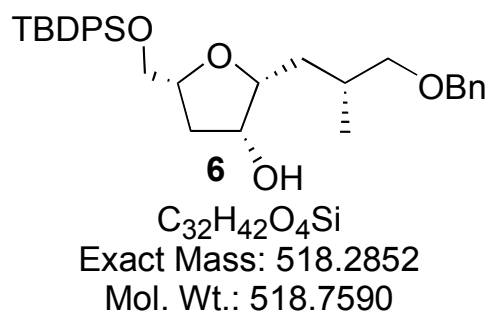

tert-Butyldiphenysilyl Ether 6. To a magnetically stirred, rt solution of diol $\mathbf{5}^{1}(68.4 \mathrm{mg}$, $244 \mu \mathrm{mol})$ in $\mathrm{CH}_{2} \mathrm{Cl}_{2}(3 \mathrm{~mL})$ was added TBDPSCl $(80 \mu \mathrm{L}, 0.29 \mathrm{mmol})$ and imidazole (33.2 $\mathrm{mg}, 488 \mu \mathrm{mol})$. The mixture was stirred for $12 \mathrm{~h}$ before satd. aq. $\mathrm{NH}_{4} \mathrm{Cl}(3 \mathrm{~mL})$ was added. The separated aqueous phase was extracted with $\mathrm{CH}_{2} \mathrm{Cl}_{2}$ and the combined organic phase was dried with $\mathrm{Na}_{2} \mathrm{SO}_{4}$, filtered, and concentrated. The residue was purified by flash column chromatography (hexanes : ethyl acetate, $4: 1, \mathrm{v} / \mathrm{v})$ to give $\mathbf{5}$ as an oil $(88.6 \mathrm{mg}, 171 \mu \mathrm{mol}$, 70\%): ${ }^{1} \mathrm{H}$ NMR $(300 \mathrm{MHz}) \delta 7.82(\mathrm{~d}, J=6.6 \mathrm{~Hz}, 1 \mathrm{H}), 7.81(\mathrm{~d}, J=7.8 \mathrm{~Hz}, 1 \mathrm{H}), 7.75(\mathrm{~d}, J=$ $5.4 \mathrm{~Hz}, 1 \mathrm{H}), 7.75(\mathrm{~d}, J=7.8 \mathrm{~Hz}, 1 \mathrm{H}), 7.40(\mathrm{~m}, 6 \mathrm{H}), 4.59(\mathrm{~d}, J=12.0 \mathrm{~Hz}, 1 \mathrm{H}), 4.55(\mathrm{~d}, J=$ $12.9 \mathrm{~Hz}, 1 \mathrm{H}), 4.17(\mathrm{~m}, 1 \mathrm{H}), 4.07$ (ddd, $J=2.4,5.1,11.4 \mathrm{~Hz}, 1 \mathrm{H}), 3.90(\mathrm{~d}, J=10.8 \mathrm{~Hz}, 1 \mathrm{H})$, $3.85(\mathrm{~m}, 2 \mathrm{H}), 3.52(\mathrm{dd}, J=5.7,9.3 \mathrm{~Hz}, 1 \mathrm{H}), 3.51(\mathrm{~m}, 1 \mathrm{H}), 3.37(\mathrm{dd}, J=7.2,9.3 \mathrm{~Hz}, 1 \mathrm{H})$, 2.41 (ddd, $J=5.1,10.5,14.1 \mathrm{~Hz}, 1 \mathrm{H}), 2.12(\mathrm{~m}, 2 \mathrm{H}), 1.96$ (ddd, $J=5.7,8.4,13.8 \mathrm{~Hz}, 1 \mathrm{H})$, $1.62(\mathrm{ddd}, J=5.4,8.4,13.8 \mathrm{~Hz}, 1 \mathrm{H}), 1.11(\mathrm{~s}, 9 \mathrm{H}), 1.09(\mathrm{~d}, J=6.6 \mathrm{~Hz}, 3 \mathrm{H}) ;{ }^{13} \mathrm{C} \mathrm{NMR}(75$ $\mathrm{MHz}) \delta 138.8,135.7,135.6,132.5,132.1,129.9,129.8,128.2,127.8,127.8,127.4,127.3$, 82.1, 76.6, 76.3, 72.9, 72.4, 66.2, 37.3, 33.2, 31.0, 26.7, 19.0, 17.5; IR (neat) 3445, 1590, 1112, 1029; HRMS calc. for $\mathrm{C}_{32} \mathrm{H}_{42} \mathrm{O}_{4} \mathrm{SiNa}(\mathrm{M}+\mathrm{Na}) 541.2750$, found 541.2736 ; $[\alpha]_{\mathrm{D}}{ }^{25}-45$ (c $1.71, \mathrm{CHCl}_{3}$ ).

\footnotetext{
${ }^{1}$ Dounay, A. B.; Florence, G. J.; Saito, A.; Forsyth, C. J. Tetrahedron 2002, 58, 1868-1874.
} 


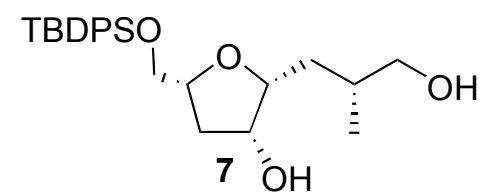

Diol 7. To a magnetically stirred, rt solution of benzyl ether 6 (130.5 mg, $252 \mu \mathrm{mol})$ in ethyl acetate $(5 \mathrm{~mL})$ was added $\mathrm{Pd}(\mathrm{OH})_{2}$ on carbon $(13 \mathrm{mg}, 20 \%$ wt. $\mathrm{Pd})$. The resulting suspension was stirred under $1 \mathrm{~atm}$ of $\mathrm{H}_{2}$ for $12 \mathrm{~h}$, at which time TLC showed no remaining 6 . The flask was flushed with $\mathrm{N}_{2}$ and the suspension was filtered through celite and $\mathrm{MgSO}_{4}$ along with ethyl acetate and ethanol risnses. Concentration of the filtrate yielded 7 (93 $\mathrm{mg}, 86 \%$ yield) as a colorless oil: ${ }^{1} \mathrm{H}$ NMR $(300 \mathrm{MHz})$ a copy of the spectrum is included below; ${ }^{13} \mathrm{C}$ NMR (75 MHz) a copy of the spectrum is included below; IR (neat) 3435, 1590, 1112, 1032; HRMS calc. for $\mathrm{C}_{25} \mathrm{H}_{36} \mathrm{O}_{4} \mathrm{Na}(\mathrm{M}+\mathrm{Na}) 451.2281$, found 451.2300.

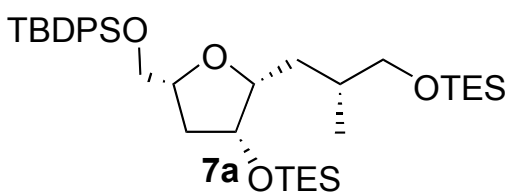

Bis-Triethylsilyl Ether 7a. To a magnetically stirred, rt solution of diol 7 (115.9 mg, 270 $\mu \mathrm{mol})$ in $\mathrm{CH}_{2} \mathrm{Cl}_{2}(3 \mathrm{~mL})$ was added imidazole $(55.2 \mathrm{mg}, 811 \mu \mathrm{mol})$, TESCl $(0.13 \mathrm{~mL}, 757$ $\mu \mathrm{mol}$ ), and DMAP (ca. $1 \mathrm{mg}$ ). The mixture was stirred for $1 \mathrm{~d}$ before satd. aq. $\mathrm{NH}_{4} \mathrm{Cl}(3 \mathrm{~mL})$ was added. The separated aqueous phase was extracted with $\mathrm{CH}_{2} \mathrm{Cl}_{2}$ and the combined organic phase was dried with $\mathrm{Na}_{2} \mathrm{SO}_{4}$, filtered, and concentrated. The residue was purified by flash column chromatography (hexanes : ethyl acetate, 20:1, v/v) to give 7a (153 mg, 232 $\mu \mathrm{mol}, 83 \%$ ) as an oil: ${ }^{1} \mathrm{H}$ NMR $(300 \mathrm{MHz})$ a copy of the spectrum is included below; ${ }^{13} \mathrm{C}$ NMR $(75 \mathrm{MHz})$ a copy of the spectrum is included below; IR (neat) 2955, 2877, 1590, 1461, 1109, 1012; HRMS calc. for $\mathrm{C}_{37} \mathrm{H}_{64} \mathrm{O}_{4} \mathrm{Si}_{3} \mathrm{Na}(\mathrm{M}+\mathrm{Na}) 679.4010$, found 679.3990; $[\alpha]_{\mathrm{D}}{ }^{25}-8.2$ (c $0.87, \mathrm{CHCl}_{3}$ ).

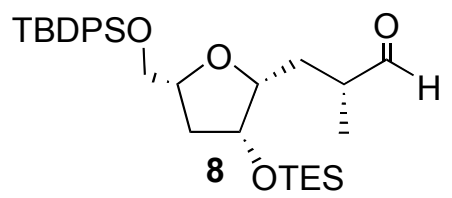

Aldehyde 8. To a 2-neck $5 \mathrm{ml}$ flask with an internal thermometer was added $\mathrm{CH}_{2} \mathrm{Cl}_{2}(0.5$ $\mathrm{mL})$ and oxalyl chloride $(24 \mu \mathrm{L}, 274 \mu \mathrm{mol})$. The magnetically stirred solution was cooled to $-78^{\circ} \mathrm{C}$ before DMSO (40 $\mu \mathrm{L}, 0.55 \mathrm{mmol}$ ) was added. After stirring for $30 \mathrm{~min}$, a solution of $7 \mathbf{a}(45.1 \mathrm{mg}, 68.5 \mu \mathrm{mol})$ in $\mathrm{CH}_{2} \mathrm{Cl}_{2}$ (ca. $0.2 \mathrm{~mL}$ ) was added and stirring was continued at $78^{\circ} \mathrm{C}$ for $30 \mathrm{~min}$. The mixture was warmed to $-35^{\circ} \mathrm{C}$ and held at that temperature for $3 \mathrm{~min}$ before being re-cooled to $-78^{\circ} \mathrm{C}$. Diisopropylethylamine $(0.16 \mathrm{~mL}, 0.9 \mathrm{mmol})$ was added dropwise and the resulting solution was stirred at $-78^{\circ} \mathrm{C}$ for $30 \mathrm{~min}$ before being warmed to $0^{\circ}$ C. Satd. aq. $\mathrm{NH}_{4} \mathrm{Cl}(1 \mathrm{~mL})$ was added, the separated aqueous phase was extracted with $\mathrm{CH}_{2} \mathrm{Cl}_{2}$ and the combined organic phase was dried with $\mathrm{Na}_{2} \mathrm{SO}_{4}$, filtered, and concentrated. The residue was purified by flash column chromatography (hexanes : ethyl acetate, 15:1, v/v) 
to give $8(26.9 \mathrm{mg}, 50 \mu \mathrm{mol}, 73 \%)$ and as an oil: ${ }^{1} \mathrm{H}$ NMR $(300 \mathrm{MHz})$ a copy of the spectrum is included below; ${ }^{13} \mathrm{C}$ NMR $(75 \mathrm{MHz})$ a copy of the spectrum is included below; IR (neat) 2956, 2877, 2859, 1726, 1461, 1428, 1111; HRMS calc. for $\mathrm{C}_{31} \mathrm{H}_{48} \mathrm{O}_{4} \mathrm{Si}_{2} \mathrm{Na}$ $(\mathrm{M}+\mathrm{Na}) 563.2989$, found 563.3005; $[\alpha]_{\mathrm{D}}{ }^{25}-24.1\left(c 1.345, \mathrm{CHCl}_{3}\right)$.

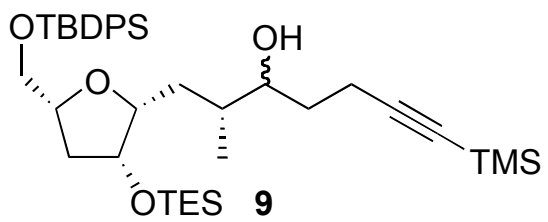

Alkynyl Alcohols 9. To a $-78^{\circ} \mathrm{C}$ solution of 4-iodo-1-trimethysilyl-1-butyne ${ }^{2}(67.8 \mathrm{mg}, 269$ $\mu \mathrm{mol})$ in diethyl ether $(1 \mathrm{~mL}, 0.27 \mathrm{M})$ was added dropwise a solution of $t$-BuLi $(0.36 \mathrm{~mL}$ of $1.7 \mathrm{M}$ in hexanes, $618 \mu \mathrm{mol})$. The solution was stirred at $-78{ }^{\circ} \mathrm{C}$ for $15 \mathrm{~min}$ then warmed to $\mathrm{rt}$ over $30 \mathrm{~min}$. The solution became clear and dark yellow. It was re-cooled to $-78{ }^{\circ} \mathrm{C}$ then cannulated into a flask containing $\mathrm{CuI}(25.6 \mathrm{mg}, 135 \mu \mathrm{mol})$ in diethyl ether $(1 \mathrm{~mL})$ at $-78{ }^{\circ} \mathrm{C}$. The mixture was stirred for $5 \mathrm{~min}$, by which time it turned green. A $-78{ }^{\circ} \mathrm{C}$ solution of aldehyde $12(14.4 \mathrm{mg}, 27 \mu \mathrm{mol})$ in diethyl ether $(1 \mathrm{~mL})$ was added and the resultant solution was stirred at $-78{ }^{\circ} \mathrm{C}$ for $1 \mathrm{~h}$. Saturated aqueous $\mathrm{NH}_{4} \mathrm{Cl}$ was added and the mixture was extracted with diethyl ether. The combined organic phase was washed with brine, dried with $\mathrm{MgSO}_{4}$, filtered and concentrated. Purification by flash column chromatography (hexanes : ethyl acetate, 4:1, v/v) gave 9 as an oil and a 1:1 mixture of diastereomers $(11.5 \mathrm{mg}, 17$ umol, 65\% yield): ${ }^{1} \mathrm{H}$ NMR $(300 \mathrm{MHz}) \delta 7.68(\mathrm{~d}, J=6.9 \mathrm{~Hz}, 8 \mathrm{H}), 7.40(\mathrm{~m}, 12 \mathrm{H}), 4.20(\mathrm{~m}$, $2 \mathrm{H}), 4.06(\mathrm{~m}, 2 \mathrm{H}), 3.80(\mathrm{~m}, 3 \mathrm{H}), 3.63(\mathrm{~m}, 3 \mathrm{H}), 3.46(\mathrm{~m}, 2 \mathrm{H}), 3.27(\mathrm{bs}, 1 \mathrm{H}), 3.03(\mathrm{bs}, 1 \mathrm{H})$, $2.38(\mathrm{~m}, 3 \mathrm{H}), 2.17(\mathrm{~m}, 3 \mathrm{H}), 1.70(\mathrm{~m}, 10 \mathrm{H}), 1.44(\mathrm{~m}, 2 \mathrm{H}), 1.26(\mathrm{~s}, 4 \mathrm{H}), 1.06(\mathrm{~s}, 18 \mathrm{H}), 0.93(\mathrm{t}$, $J=8.1 \mathrm{~Hz}, 18 \mathrm{H}), 0.55(\mathrm{q}, J=7.5 \mathrm{~Hz}, 12 \mathrm{H}), 0.14(\mathrm{~s}, 9 \mathrm{H}), 0.14(\mathrm{~s}, 9 \mathrm{H}) ;{ }^{13} \mathrm{C} \mathrm{NMR}(75 \mathrm{MHz}) \delta$ $135.5,133.6,129.4,127.5,107.8,84.2,82.4,81.9,78.2,78.0,75.2,73.3,73.2,66.8,38.1$, 38.0, 37.4, 37.1, 34.5, 33.2, 33.0, 32.2, 29.5, 26.6, 19.0, 17.2, 17.1, 16.5, 15.6, 6.6, 4.6, 0.0; IR (neat) 3419, 2173, 1111; HRMS calc. for $\mathrm{C}_{32} \mathrm{H}_{47} \mathrm{O}_{3} \mathrm{Si}_{2}(\mathrm{M}+\mathrm{Na})$ 689.3852, found 689.3877 .

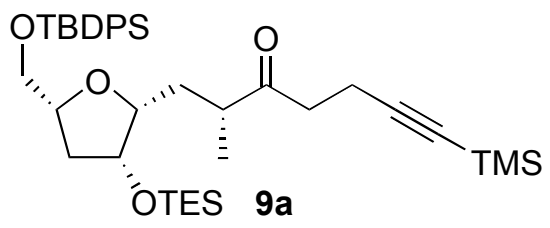

Ketone 9a. To a stirred rt solution of alcohol $9(55.7 \mathrm{mg}, 104 \mu \mathrm{mol})$ in $\mathrm{CH}_{2} \mathrm{Cl}_{2}(2 \mathrm{~mL})$ was added crushed $4 \AA$ mol. sieves (ca. $100 \mathrm{mg}$ ), NMO (24.4 mg, $21 \mu \mathrm{mol}$ ), and TPAP (2 mg, 5 $\mu \mathrm{mol})$. The mixture was stirred at $\mathrm{rt}$ until no 9 remained (TLC), then it was filtered through a small pad of silica gel along with ethyl acetate washes. The combined organic filtrate was concentrated, and the residue purified by flash column chromatography (hexanes : ethyl acetate, $4: 1, \mathrm{v} / \mathrm{v})$ to give 9a as an oil: ${ }^{1} \mathrm{H}$ NMR $(300 \mathrm{MHz}) \delta 7.69(\mathrm{~m}, 5 \mathrm{H}), 7.40(\mathrm{~m}, 5 \mathrm{H})$, $4.23(\mathrm{~m}, 1 \mathrm{H}), 4.01(\mathrm{~m}, 1 \mathrm{H}), 3.78$ (dd, $J=5.7 \mathrm{~Hz}, 9.9 \mathrm{~Hz}, 1 \mathrm{H}), 3.71$ (ddd, $J=3.9 \mathrm{~Hz}, 3.9 \mathrm{~Hz}$,

\footnotetext{
${ }^{2}$ Wei, X.; Taylor, R. J. K. Angew. Chem. 2000, 39, 409-412.
} 
$9.3 \mathrm{~Hz}, 1 \mathrm{H}), 3.63$ (dd, $J=9 \mathrm{~Hz}, 9.9 \mathrm{~Hz}, 1 \mathrm{H}), 3,3 \mathrm{H}), 2.67$ (m, 1H), 2.43 (m, 2H), 2.19 (ddd, $J$ $=5.7 \mathrm{~Hz}, 7.8 \mathrm{~Hz}, 13.5 \mathrm{~Hz}, 1 \mathrm{H}), 1.98$ (ddd, $J=6.3 \mathrm{~Hz}, 9.6 \mathrm{~Hz}, 14.4 \mathrm{~Hz}, 1 \mathrm{H}), 1.80$ (ddd, $J=3$ $\mathrm{Hz}, 85.7 \mathrm{~Hz}, 13.5 \mathrm{~Hz}, 1 \mathrm{H}), 1.46(\mathrm{ddd}, J=3.9 \mathrm{~Hz}, 8.4 \mathrm{~Hz}, 14.1 \mathrm{~Hz}, 1 \mathrm{H}), 1.10$ (d, $J=6.9 \mathrm{~Hz}$, $3 \mathrm{H}), 1.06(\mathrm{~s}, 9 \mathrm{H}), 0.93(\mathrm{t}, J=7.8 \mathrm{~Hz}, 9 \mathrm{H}), 0.57(\mathrm{~m}, 6 \mathrm{H}), 0.13(\mathrm{~s}, 9 \mathrm{H}) ;{ }^{13} \mathrm{C} \mathrm{NMR}(75 \mathrm{MHz}) \delta$ 212.4, 135.6, 133.8, 129.5, 127.6, 106.2, 84.6, 80.4, 78.0, 72.8, 67.1, 43.4, 39.6, 38.3, 32.8, $31.9,29.7,26.8,22.7,19.3,15.9,14.4,14.1,6.8,4.7,0.1$; IR (neat) 2177, 1715, 1110; HRMS calc. for $\mathrm{C}_{32} \mathrm{H}_{45} \mathrm{O}_{3} \mathrm{Si}_{2}(\mathrm{M}+\mathrm{Na}) 687.3691$, found $687.3663 ;[\alpha]_{\mathrm{D}}{ }^{25}-3.5(c=0.75$, $\left.\mathrm{CHCl}_{3}\right)$.

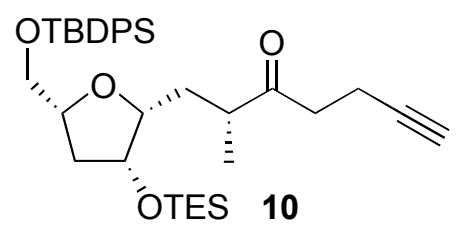

Ketone 10. To a magnetically stirred, rt solution of 9a $(15 \mathrm{mg}, 22 \mu \mathrm{mol})$ in ethanol $(2.0 \mathrm{~mL})$ and $\mathrm{H}_{2} \mathrm{O}(0.5 \mathrm{~mL})$ was added $\mathrm{AgNO}_{3}(4.2 \mathrm{mg}, 25 \mu \mathrm{mol})$. After TLC indicated that 9a $\left(\mathrm{R}_{\mathrm{f}} 0.8\right.$, $4: 1 \mathrm{v} / \mathrm{v}$, hexanes : ethyl acetate) was consumed $\left(\mathrm{R}_{\mathrm{f}} 0,4: 1 \mathrm{v} / \mathrm{v}\right.$, hexanes : ethyl acetate), KI $(4.8 \mathrm{mg}, 29 \mu \mathrm{mol})$ was added. A yellow precipitate formed and the silver acetylide $\left(\mathrm{R}_{\mathrm{f}} 0,4: 1\right.$ $\mathrm{v} / \mathrm{v}$, hexanes : ethyl acetate) was converted into higher $\mathrm{R}_{\mathrm{f}}$ products (TES ether and the corresponding secondary alcohol). The reaction mixture was filtered through celite along with ethanol and ethyl acetate washes. The filtrate was concentrated and subjected to triethylsilylation as follows.

The crude product was dissolved in $\mathrm{CH}_{2} \mathrm{Cl}_{2}(0.5 \mathrm{~mL})$ and to the $\mathrm{rt}$, magnetically stirred solution was added imidazole $(6 \mathrm{mg}, 93 \mu \mathrm{mol})$ and TESCl $(10 \mu \mathrm{L} 46 \mu \mathrm{mol})$. The resulting mixture was stirred at $\mathrm{rt}$ for $12 \mathrm{~h}$. Saturated aqueous $\mathrm{NH}_{4} \mathrm{Cl}(1 \mathrm{~mL})$ was added, the separated aqueous phase was extracted with $\mathrm{CH}_{2} \mathrm{Cl}_{2}$ and the combined organic phase was dried with $\mathrm{Na}_{2} \mathrm{SO}_{4}$, filtered, and concentrated. The residue was purified by flash column chromatography (hexanes : ethyl acetate, 10:1, v/v) to give $\mathbf{1 0}(11.7 \mathrm{mg}, 18 \mu \mathrm{mol}, 82 \%)$ as an oil: ${ }^{1} \mathrm{H}$ NMR $(300 \mathrm{MHz})$ a copy of the spectrum is included below; ${ }^{13} \mathrm{C}$ NMR $(75 \mathrm{MHz})$ a copy of the spectrum is included below; IR (neat) 2958, 2932, 2358, 1716, 1459, 1429, 1111; HRMS calc. for $\mathrm{C}_{38} \mathrm{H}_{52} \mathrm{O}_{4} \mathrm{Si}_{2} \mathrm{Na}(\mathrm{M}+\mathrm{Na}) 615.3296$, found $615.3284 ;[\alpha]_{\mathrm{D}}{ }^{25}-5.9$ (c 0.785 , $\left.\mathrm{CHCl}_{3}\right)$.

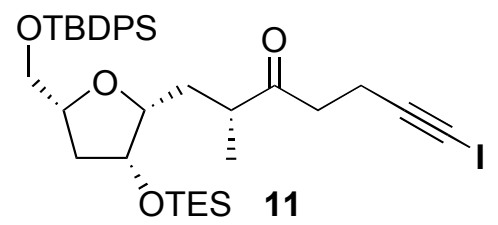

Iodide 11. To a magnetically stirred, $0{ }^{\circ} \mathrm{C}$ solution of $\mathbf{1 0}(10.0 \mathrm{mg}, 16.8 \mu \mathrm{mol})$ in acetone (1 $\mathrm{mL})$ was added silver trifluoroacetate $(1.1 \mathrm{mg}, 5 \mu \mathrm{mol})$ and $N$-iodosuccinimide $(5.7 \mathrm{mg}, 25$ $\mu \mathrm{mol})$. After stirring for $15 \mathrm{~min}$, saturated aqueous $\mathrm{Na}_{2} \mathrm{~S}_{2} \mathrm{O}_{3}(1 \mathrm{~mL})$ was added and the mixture was extracted with diethyl ether $(4 \mathrm{x} 1 \mathrm{~mL})$. The combined organic extract was dried over $\mathrm{MgSO}_{4}$, filtered, and concentrated to provide $11(12.1 \mathrm{mg})$ as a clear, colorless oil: 
$\mathrm{C}_{38} \mathrm{H}_{51} \mathrm{O}_{4} \mathrm{Si}_{2} \mathrm{NaI}(\mathrm{M}+\mathrm{Na}) 742.2264$, found 741.2270. This was used in the following reaction without further purification.

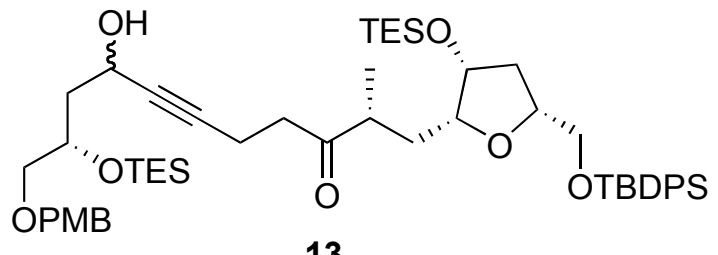

13

Propargyl Alcohols 13. A rt solution of iodide 11 (12 mg, ca. $17 \mu \mathrm{mol})$ and aldehyde $\mathbf{1 2}^{3}$ $(9.4 \mathrm{mg}, 28 \mu \mathrm{mol})$ in THF $(0.5 \mathrm{~mL})$ was transferred via cannula along with additional THF rinses to a septum capped flask containing powdered anhydrous $\mathrm{CrCl}_{2} / 1 \% \mathrm{NiCl}_{2}$ (wt/wt) (17.1 mg, $0.14 \mathrm{mmol} \mathrm{CrCl}_{2}$ ). The resulting mixture was stirred at $\mathrm{rt}$ for $12 \mathrm{~h}$. Saturated aqueous $\mathrm{NaHCO}_{3}(1 \mathrm{~mL})$ was added and the resulting mixture was extracted with diethyl ether $(4 \times 1 \mathrm{~mL})$. The combined organic extract was dried over $\mathrm{MgSO}_{4}$, fitered, and concentrated. The residue was purified by flash silica gel column chromatography (hexanes : ethyl acetate, $4: 1 \mathrm{v} / \mathrm{v})$ to yield $13(11.4 \mathrm{mg}, 12 \mu \mathrm{mol}, 68 \%)$ as a colorless film: ${ }^{1} \mathrm{H}$ NMR (300 MHz) a copy of the spectrum is included below; ${ }^{13} \mathrm{C}$ NMR $(75 \mathrm{MHz}$ ) a copy of the spectrum is included below; IR (neat) 3400, 2958, 2924, 1729, 1714, 1445; HRMS calc. $(\mathrm{M}+\mathrm{Na})$ 953.5209, found 953.5158.

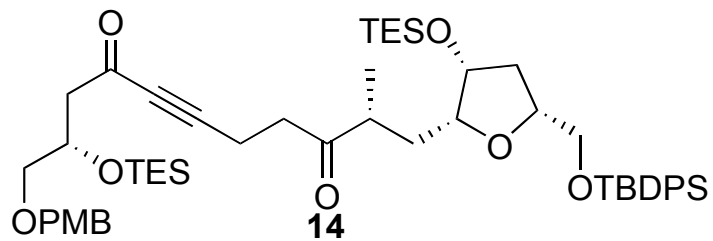

Yne-dione 14. To a solution of alcohol $13(2.7 \mathrm{mg}, 2.9 \mu \mathrm{mol})$ in $\mathrm{CH}_{2} \mathrm{Cl}_{2}(1 \mathrm{~mL})$ was added tert-butanol (ca. $10 \mu \mathrm{L})$, Dess-Martin periodinane $(3.7 \mathrm{mg}, 8.7 \mu \mathrm{mol})$ and solid $\mathrm{NaHCO}_{3}(2.2$ $\mathrm{mg}, 26 \mu \mathrm{mol})$. The mixture was stirred at $\mathrm{rt}$ for $4 \mathrm{~h}$ before additional Dess-Martin periodinane $(3.7 \mathrm{mg}, 8.7 \mu \mathrm{mol})$ and solid $\mathrm{NaHCO}_{3}(2.2 \mathrm{mg}, 26 \mu \mathrm{mol})$ were added. After an additional $4 \mathrm{~h}$, the reaction mixture was concentrated to half its volume then diluted with diethyl ether $(0.5 \mathrm{~mL})$. The mixture was loaded onto a silica gel coumn and eluted (hexanes : ethyl acetate, 5:1, v/v) to give crude diketone $\mathbf{1 4}$ as a colorless oil. This material was subjected to the next transformation without further purification. ${ }^{1} \mathrm{H} \mathrm{NMR}(300 \mathrm{MHz}) \delta 7.67$ $(\mathrm{m}, 4 \mathrm{H}), 7.39(\mathrm{~m}, 6 \mathrm{H}), 7.24(\mathrm{~d}, J=8.4 \mathrm{~Hz}, 2 \mathrm{H}), 6.89(\mathrm{~d}, J=8.4 \mathrm{~Hz}, 2 \mathrm{H}), 4.45(\mathrm{~s}, 2 \mathrm{H}), 4.40$ $(\mathrm{m}, 1 \mathrm{H}), 4.22(\mathrm{~m}, 1 \mathrm{H}), 4.00(\mathrm{~m}, 1 \mathrm{H}), 3.81(\mathrm{~s}, 3 \mathrm{H}), 3.72(\mathrm{~m}, 1 \mathrm{H}), 3.62(\mathrm{dd}, J=6.3,10.2 \mathrm{~Hz}$, $1 \mathrm{H}), 3.42(\mathrm{dd}, J=5.4,9.6 \mathrm{~Hz}, 1 \mathrm{H}), 3.32(\mathrm{dd}, J=6.0,9.6 \mathrm{~Hz}, 1 \mathrm{H}), 2.64(\mathrm{~m}, 6 \mathrm{H}), 2.19$ (ddd, $J$ $=5.7,7.5,13.2 \mathrm{~Hz}, 1 \mathrm{H}), 1.97(\mathrm{ddd}, J=6.0,9.3,15.0 \mathrm{~Hz}, 1 \mathrm{H}), 1.80(\mathrm{ddd}, J=3.0,5.7,12.9$ $\mathrm{Hz}, 1 \mathrm{H}), 1.45$ (ddd, $J=3.3,7.8,13.8 \mathrm{~Hz}, 1 \mathrm{H}), 1.10$ (d, $J=6.9 \mathrm{~Hz}, 3 \mathrm{H}), 1.05$ (s, 9H), 0.93 (m, $18 \mathrm{H}), 0.57(\mathrm{~m}, 12 \mathrm{H}), 0.08(\mathrm{~s}, 9 \mathrm{H})$; HRMS calc. for $\mathrm{C}_{41} \mathrm{H}_{50} \mathrm{O}_{6} \mathrm{Si}(\mathrm{M}+\mathrm{Na})$ 951.5053, found 951.5092 .

${ }^{3}$ Paterson, I.; Coster, M. J. Tetrahedron Lett. 2002, 43, 3285-3290. 


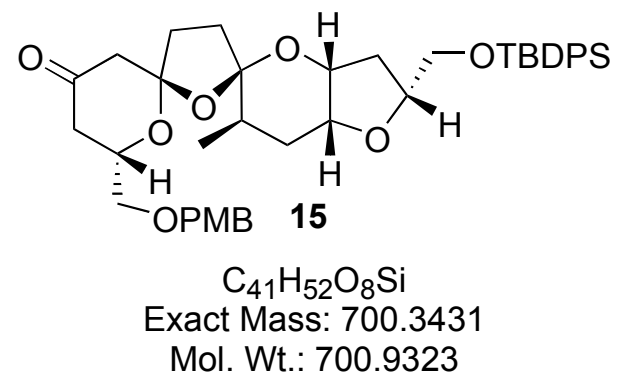

Trioxadispiroketal 15. To a magnetically stirred solution of yne-dione $\mathbf{1 4}$ in toluene $(0.4$ $\mathrm{mL}$ ) was added $p-\mathrm{TsOH}_{2} \mathrm{H}(0.5 \mathrm{mg})$ and the solution was stirred at $\mathrm{rt}$ for $1-2 \mathrm{~d}$ (completion monitored by ms). Triethylamine $(20 \mu \mathrm{L})$ was added and the mixture was concentrated. Purification of the residue by flash chromatography (hexanes : ethyl acetate, $3: 1, \mathrm{v} / \mathrm{v}$ ) gave ketone 15 (1.1 mg, 1.5 umol, 55\% yield from 13): IR (film, $\left.\mathrm{cm}^{-1}\right) 1717,1278 ;{ }^{1} \mathrm{H}$ NMR (500 MHz) $\delta 7.69(\mathrm{~m}, 4 \mathrm{H}), 7.39(\mathrm{~m}, 6 \mathrm{H}), 7.25(\mathrm{~d}, J=8.0 \mathrm{~Hz}, 2 \mathrm{H}), 6.88(\mathrm{~d}, J=9.0 \mathrm{~Hz}, 2 \mathrm{H}), 4.56$ $(\mathrm{d}, J=12.0 \mathrm{~Hz}, 1 \mathrm{H}), 4.48$ (d, $J=12.3 \mathrm{~Hz}, 1 \mathrm{H}), 4.29$ (dddd, $J=3.0,4.0,4.5,11.0 \mathrm{~Hz}, 1 \mathrm{H}$ ), $4.17(\mathrm{~m}, 1 \mathrm{H}), 4.08(\mathrm{~m}, 1 \mathrm{H}), 3.82(\mathrm{~s}, 3 \mathrm{H}), 3.75(\mathrm{~m}, 2 \mathrm{H}), 3.62(\mathrm{dd}, J=7.0,10.5 \mathrm{~Hz}, 1 \mathrm{H}), 3.56$ (dd, $J=4.0,10.5 \mathrm{~Hz}, 1 \mathrm{H}), 3.50$ (dd, $J=4.0,10.5 \mathrm{~Hz}, 1 \mathrm{H}), 2.75(\mathrm{~d}, J=15.0 \mathrm{~Hz}, 1 \mathrm{H}), 2.48$ (m, 2H), $2.35(\mathrm{~m}, 1 \mathrm{H}), 2.14(\mathrm{~m}, 4 \mathrm{H}), 1.86(\mathrm{~m}, 3 \mathrm{H}), 1.64(\mathrm{ddd}, J=3.5,14.5,14.5 \mathrm{~Hz}, 1 \mathrm{H})$, $1.45(\mathrm{~m}, 1 \mathrm{H}), 1.05(\mathrm{~s}, 9 \mathrm{H}), 0.80(\mathrm{~d}, J=7.0 \mathrm{~Hz}, 3 \mathrm{H})$; HRMS calc. for $\mathrm{C}_{41} \mathrm{H}_{52} \mathrm{O}_{8} \mathrm{Si}(\mathrm{M}+\mathrm{Na})$ 723.3326, found 723.3321; LRMS using MeOD showed no deuterium exchange of the $\mathrm{M}+\mathrm{Na}$ peak (723.3) indicating that the compound did not contain readily exchangeable protons (i.e. alcohols). 


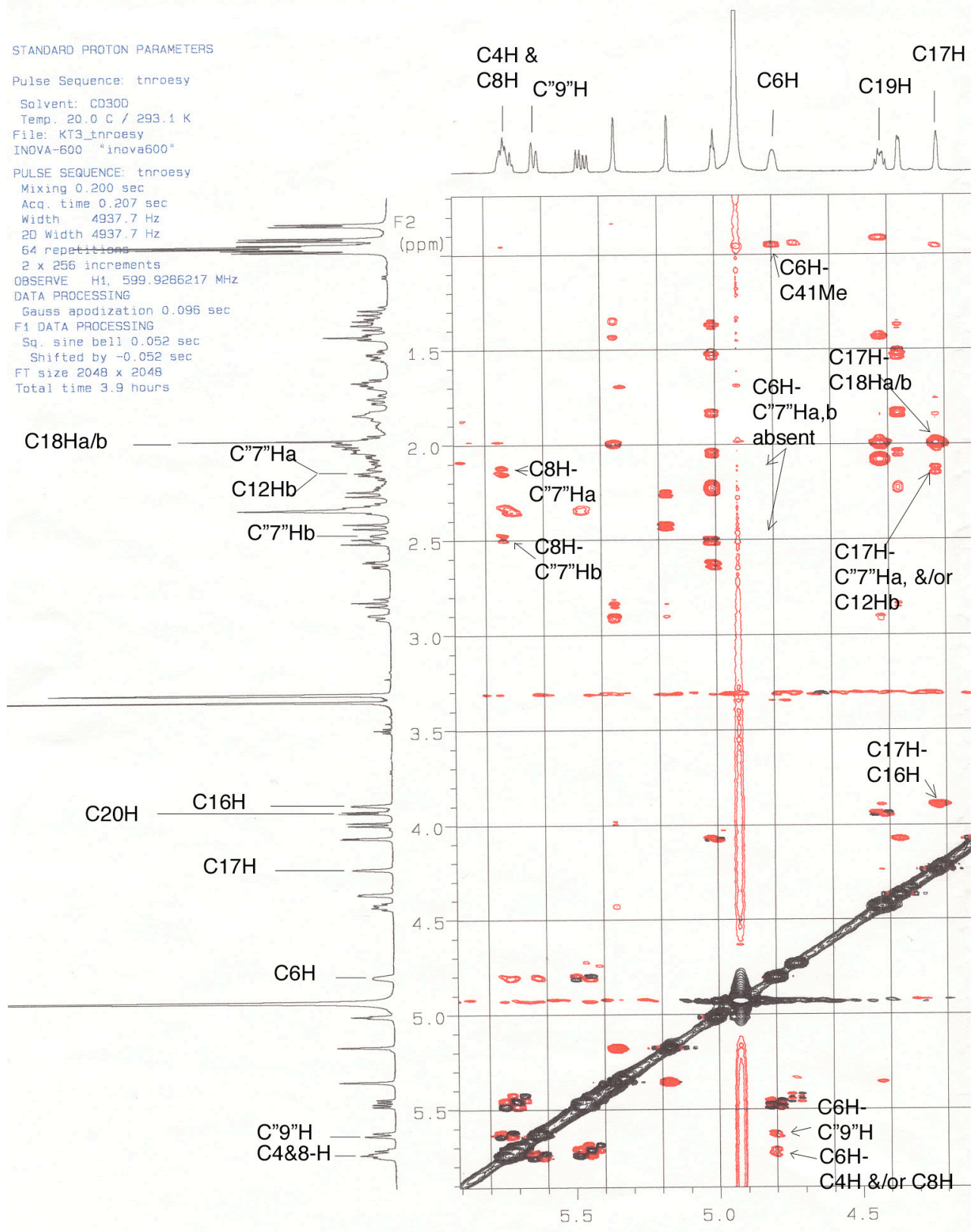

ROESY Plot of AZA-1 (kindly provided by Prof. M. Satake and annotated by C. J. Forsyth). Absent are cross peaks for $\mathrm{C} 6 \mathrm{H}$ and A-ring methylene protons $(\mathrm{C}$ "7" $\mathrm{Ha}, \mathrm{b})$; present is a cross peak for $\mathrm{C} 6 \mathrm{H}$ and the A-ring alkene proton originally assigned to C"9" $\mathrm{H}$ (the A-ring alkene is revised to C7-8). The $\mathrm{C} 17 \mathrm{H}-\mathrm{C} 12 \mathrm{Hb}$ cross peak may also be attributable to the nearer C"7"Ha (C"7"Ha is reassigned to $\mathrm{C} 9 \mathrm{Ha}$ in $\Delta^{7,8}-6,10,13$-tris-epi model). 


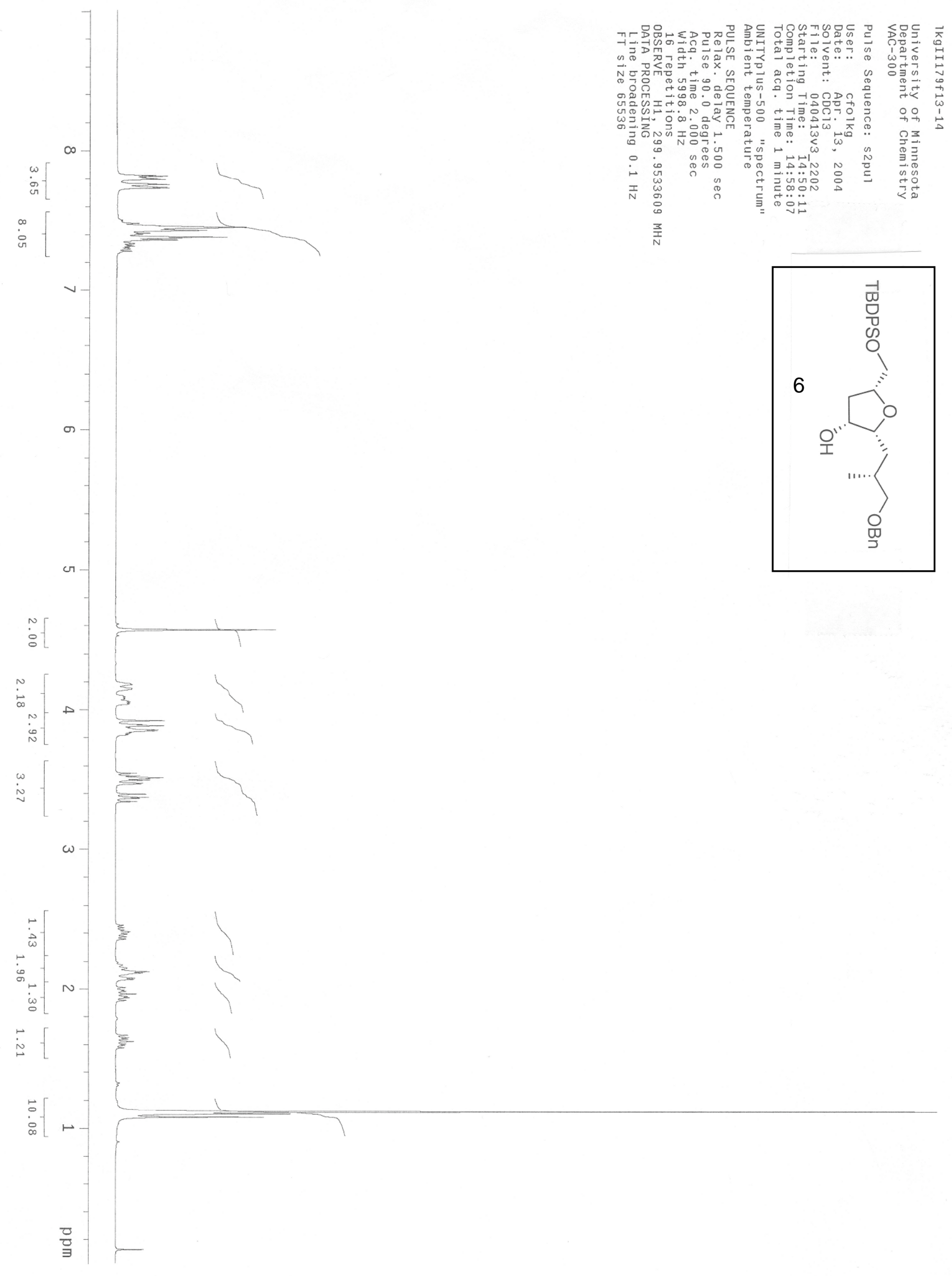




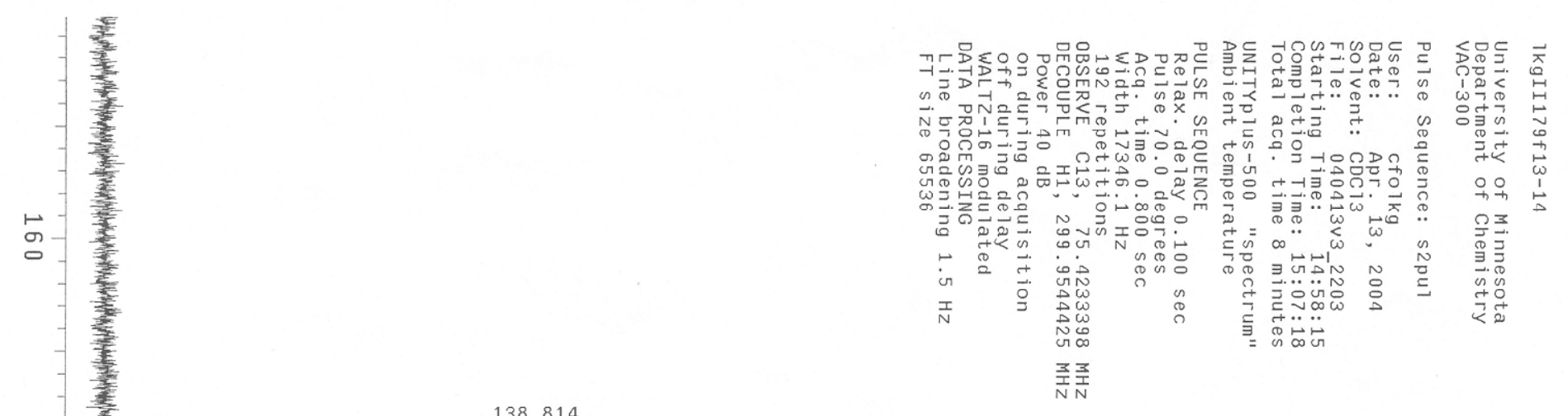

$\stackrel{b}{a}$

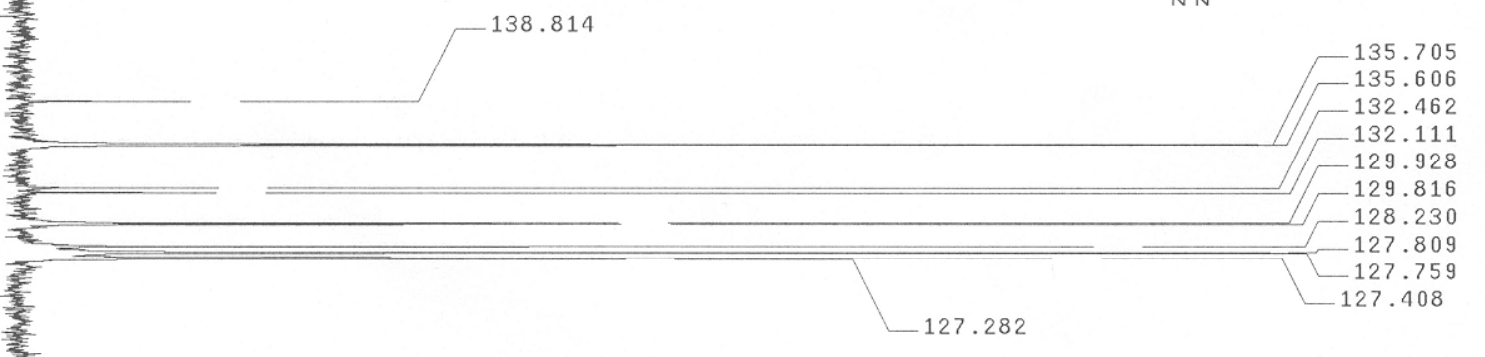

$\stackrel{\bullet}{0}$

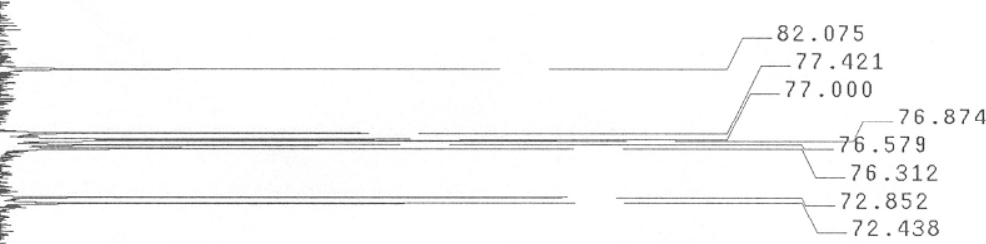

$-66.191$

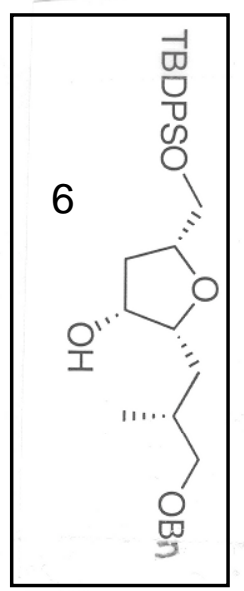

o

$-37.316$

33.231

31.027

$\sim$

-

$-19.004$ 


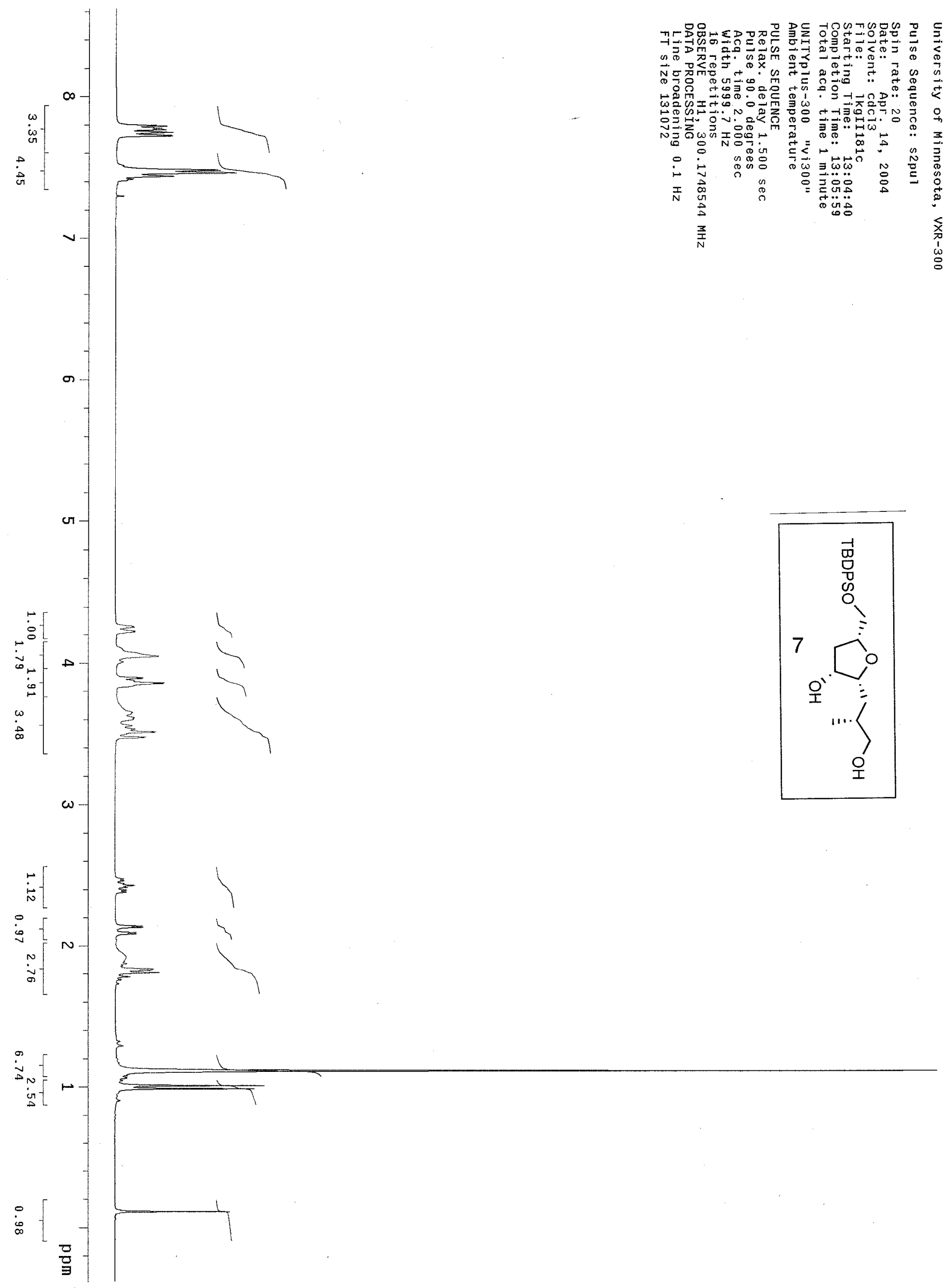




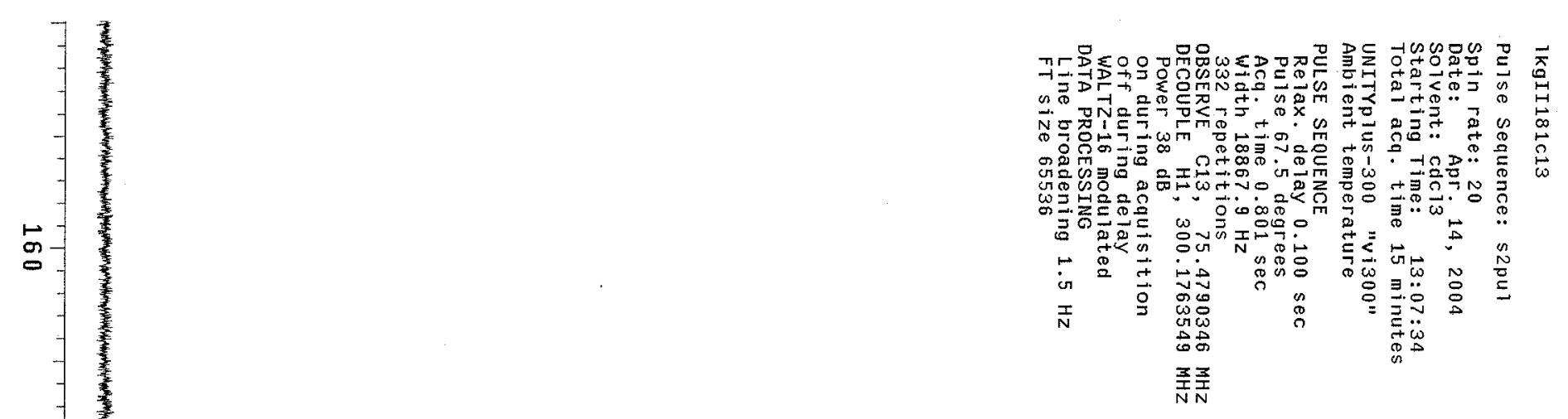

$\stackrel{b}{0}$
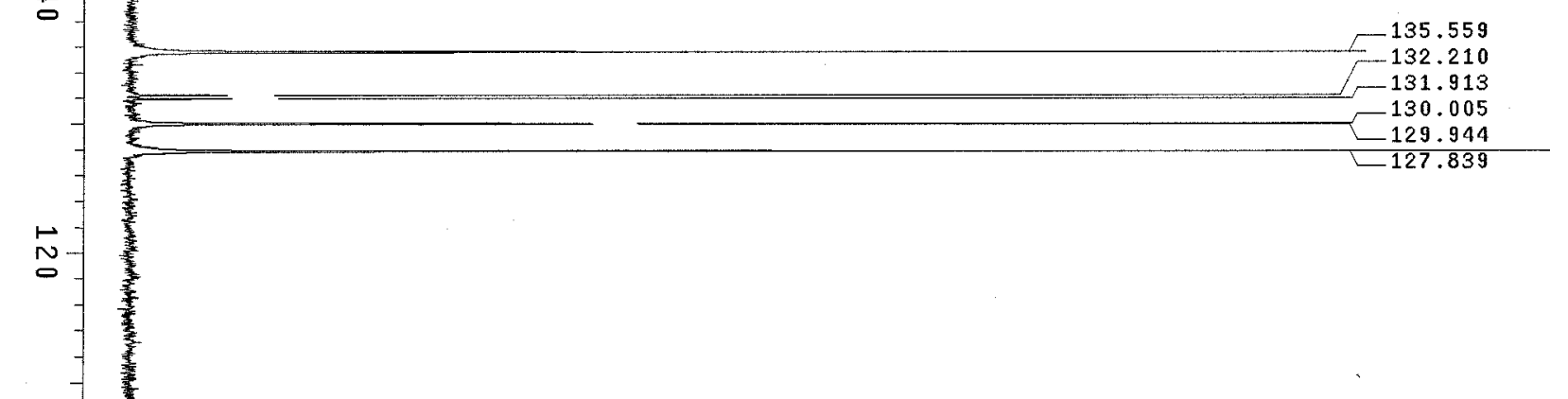

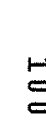

$\infty$

등-

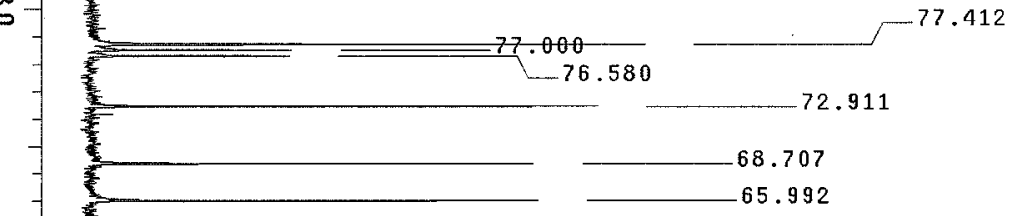

$\circ$

$\pm$


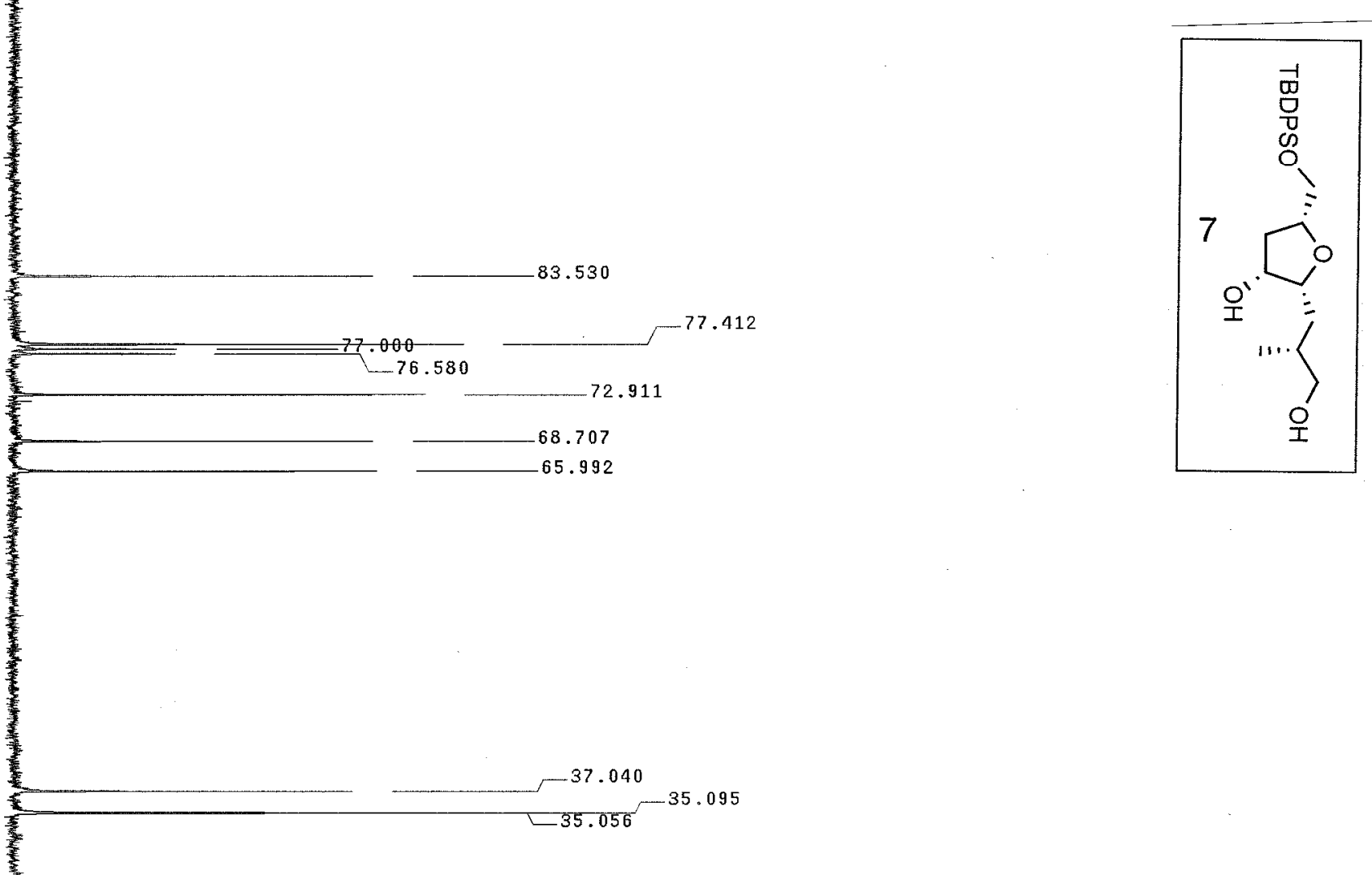

列 


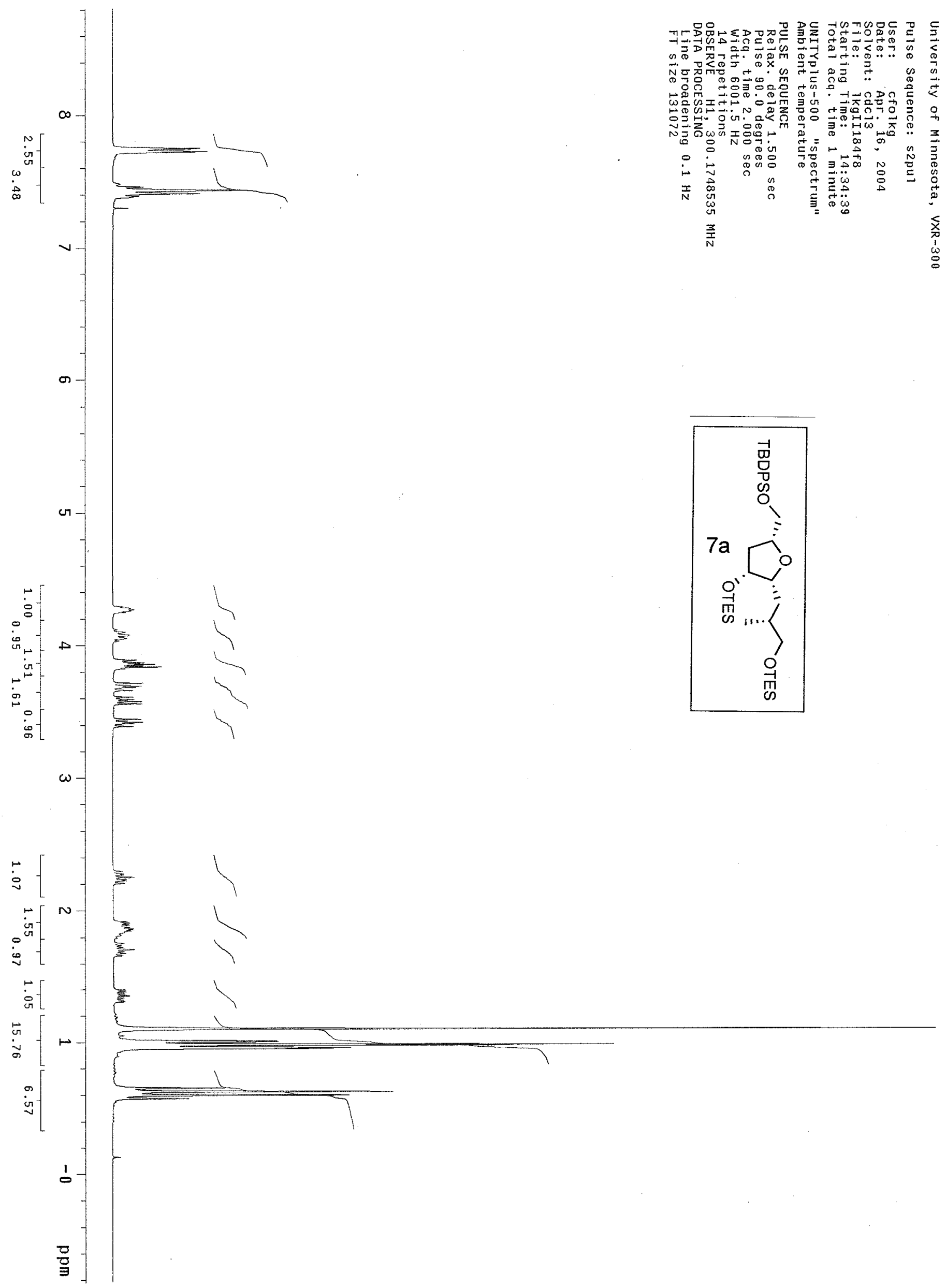




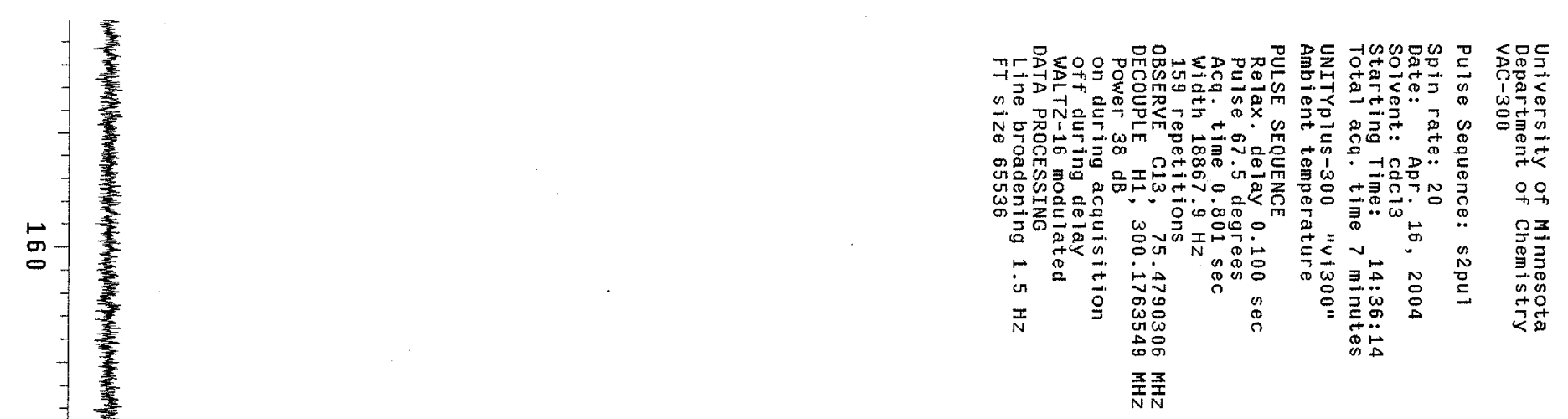

告

$\stackrel{\square}{0}$
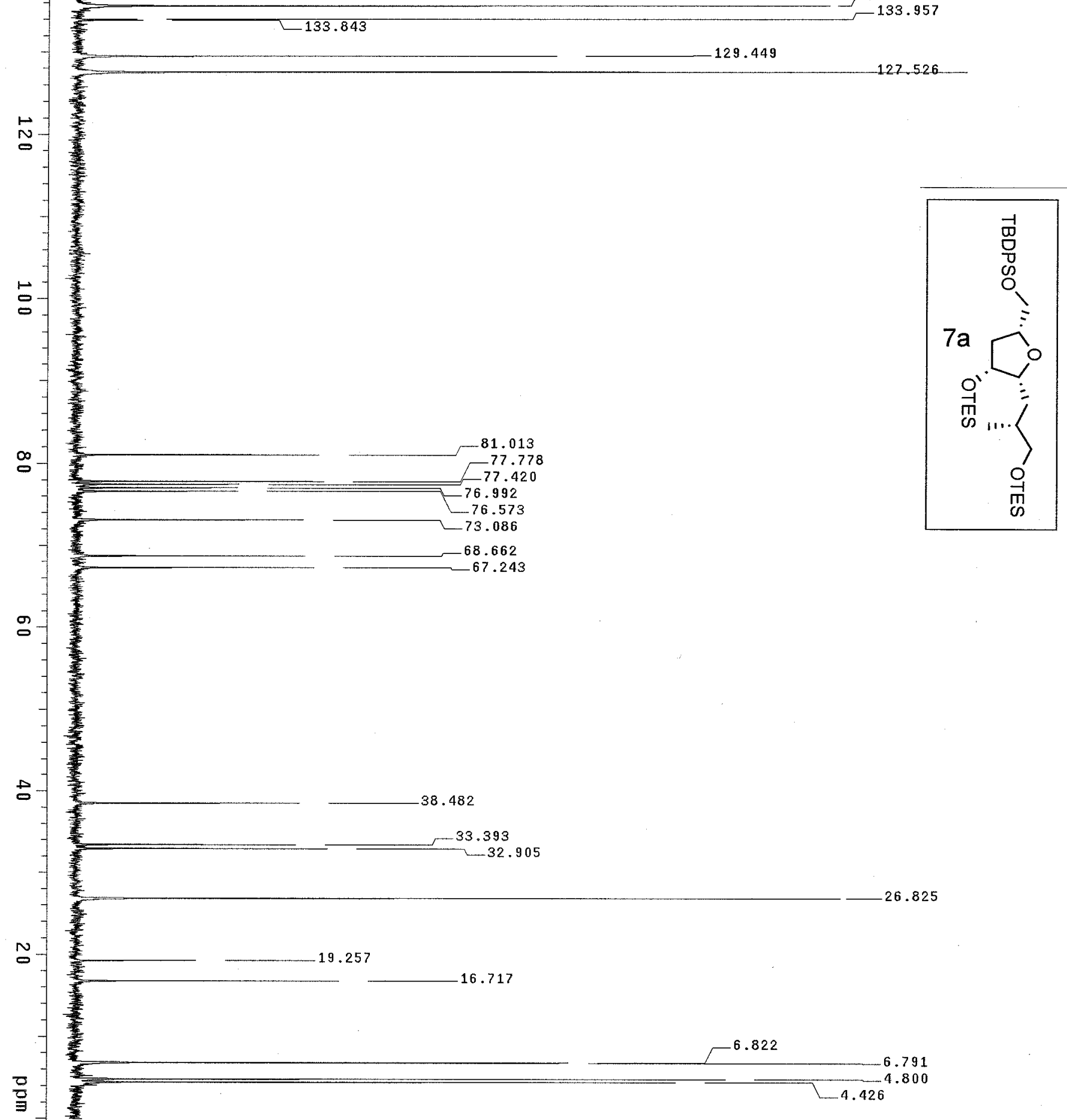

$-38.482$

33.393

$-32.905$

$-26.825$

$-16.717$

6.822 


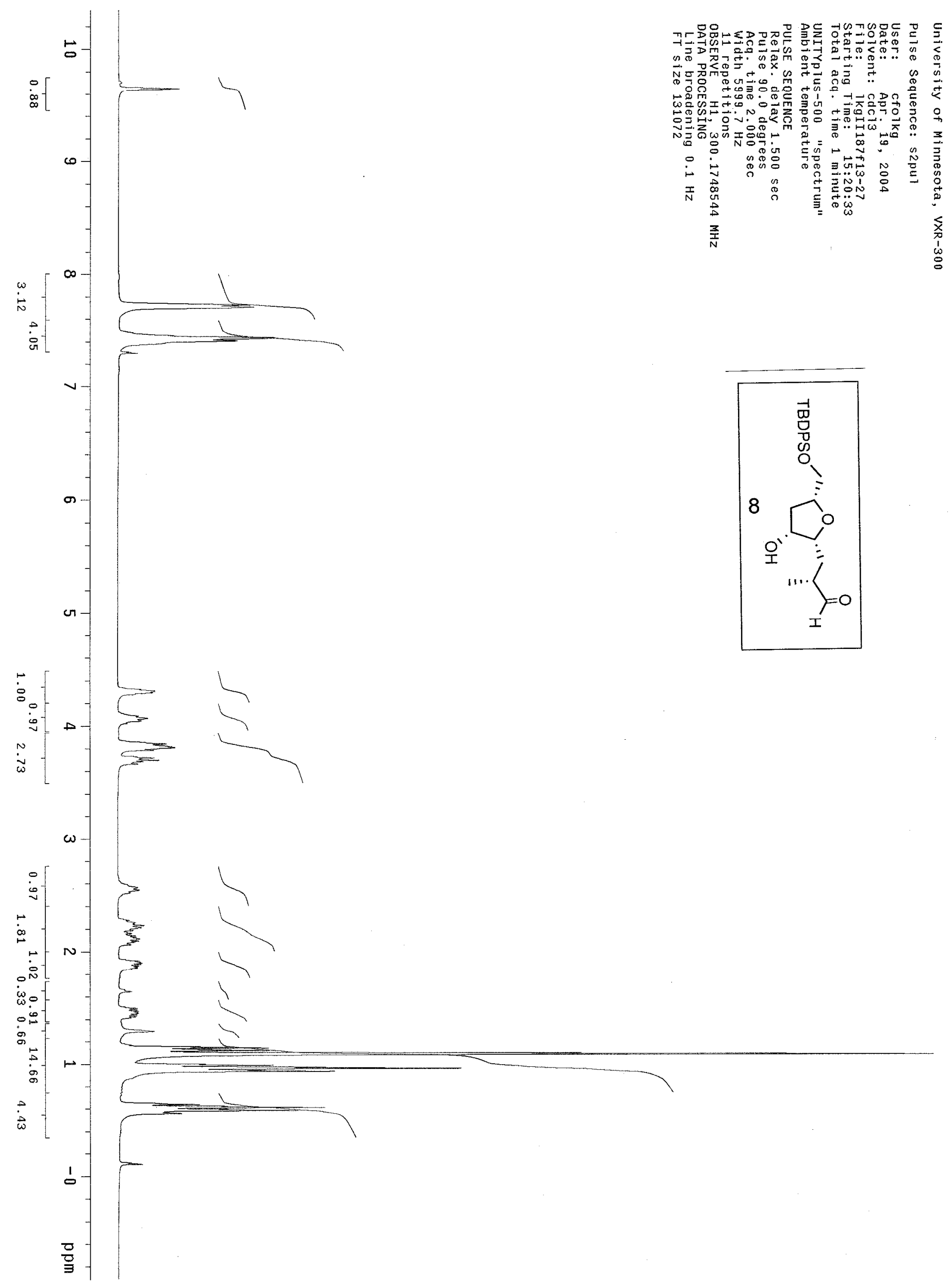



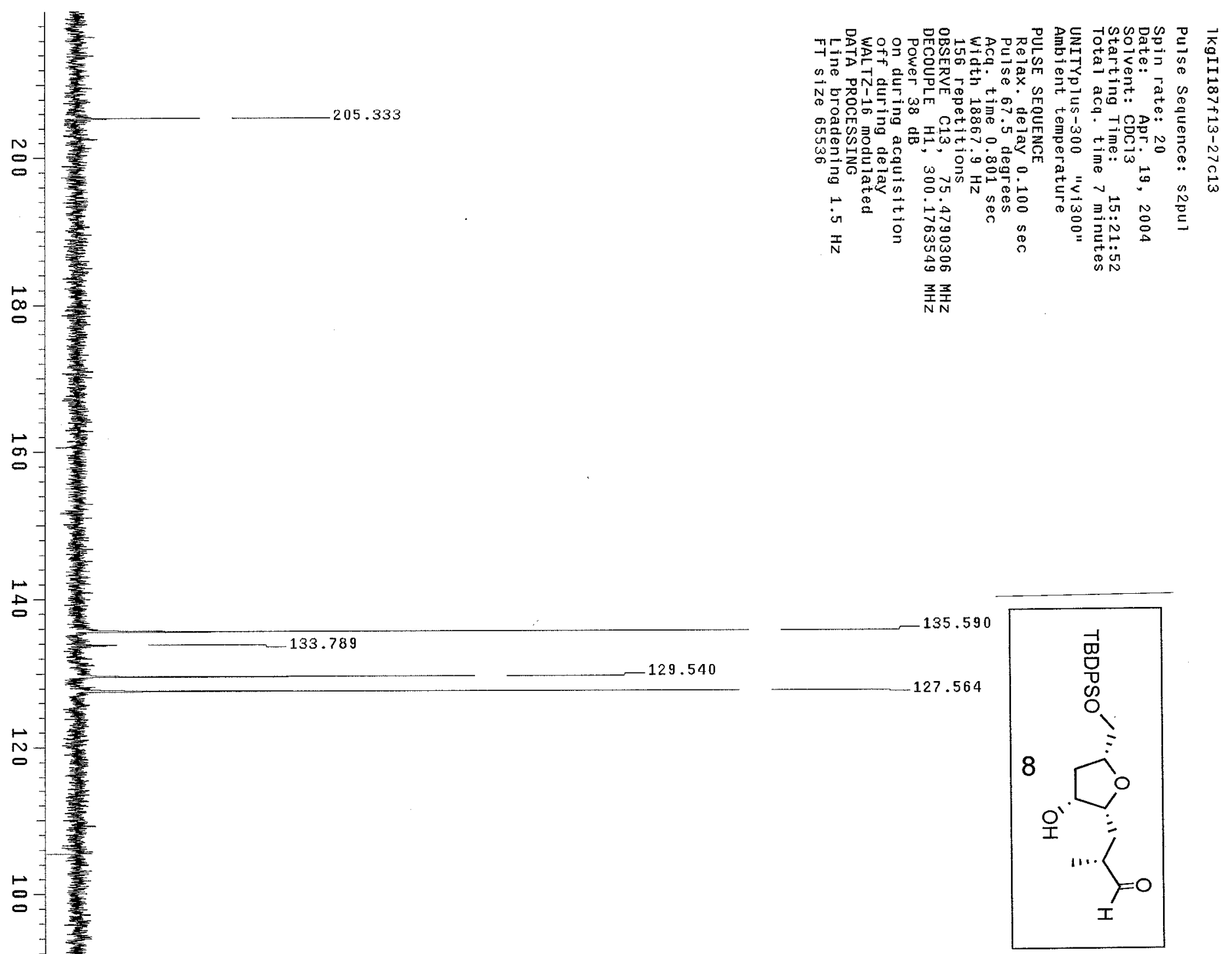

$-43.944$

$\stackrel{\circ}{\circ}$

38.070

31.044

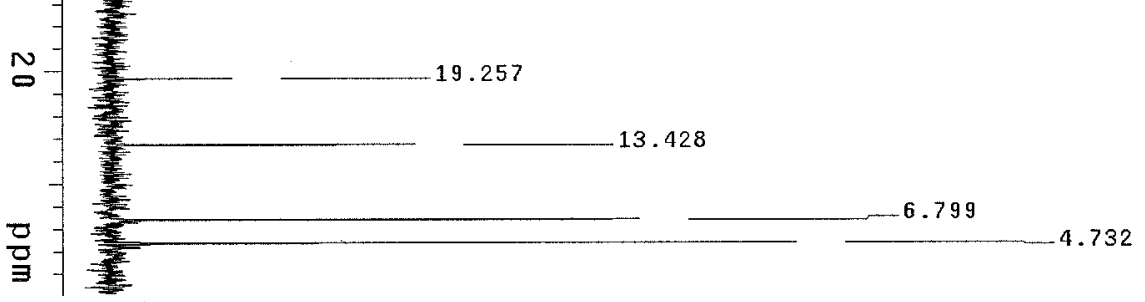

$-26.810$ 


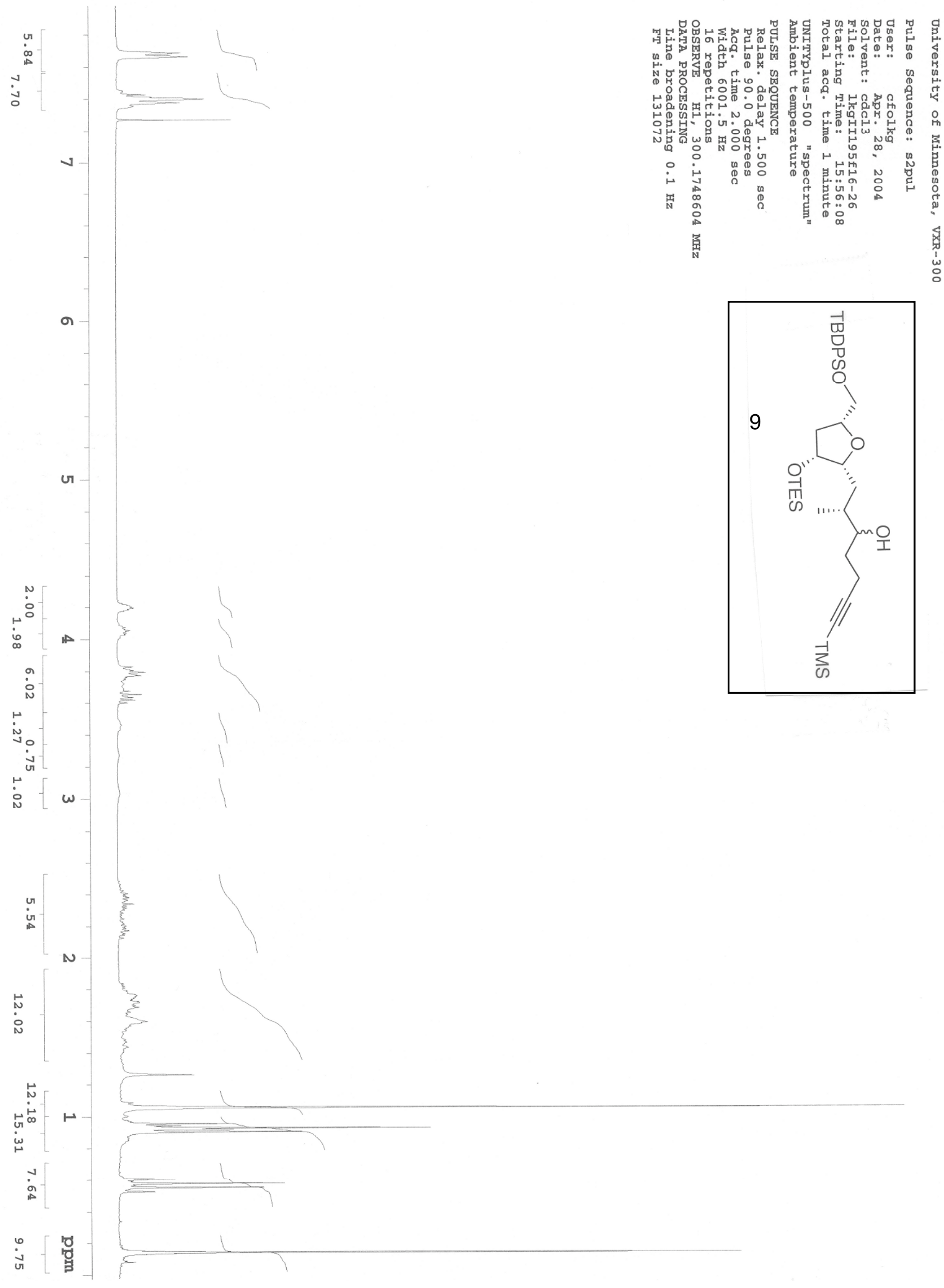




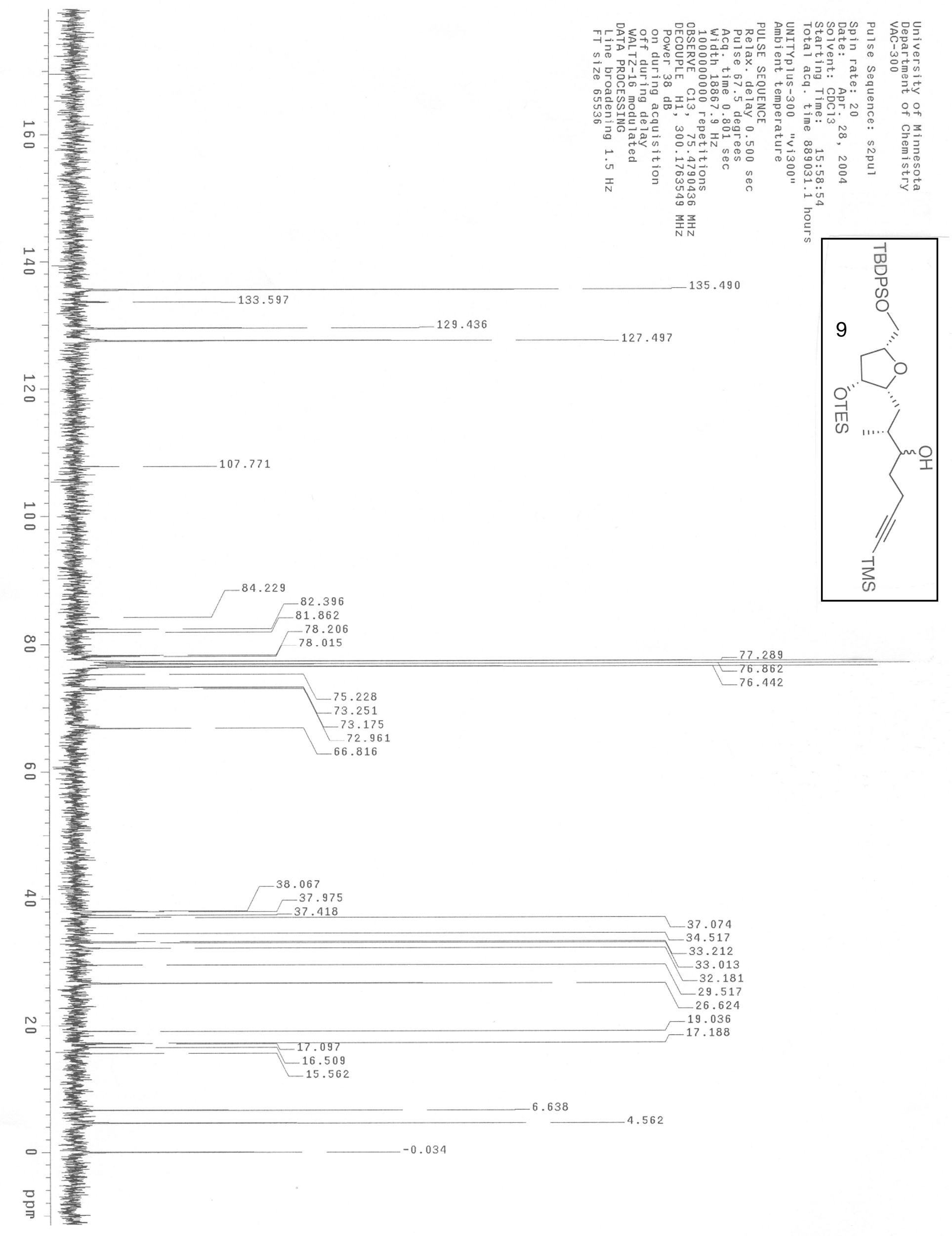



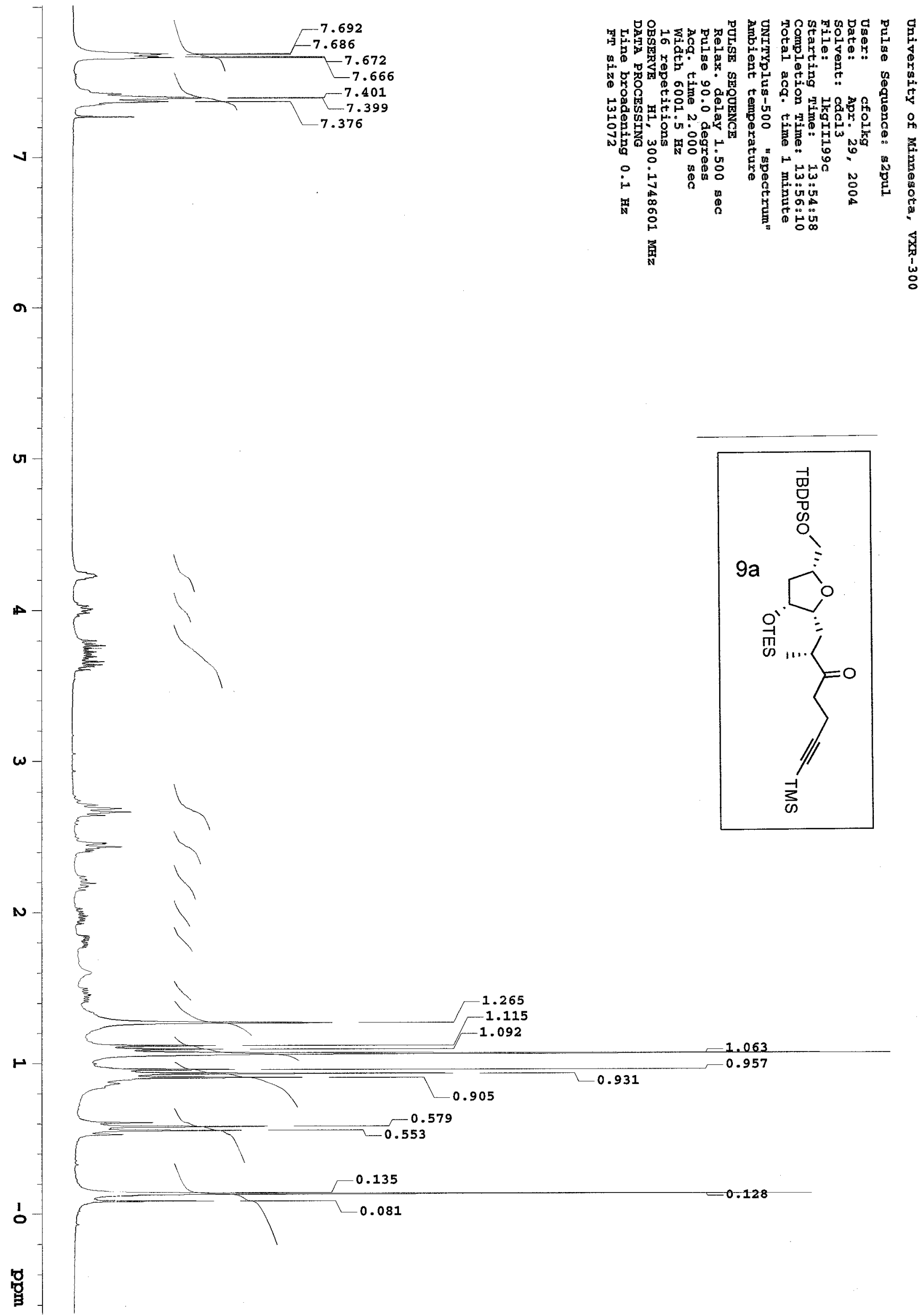


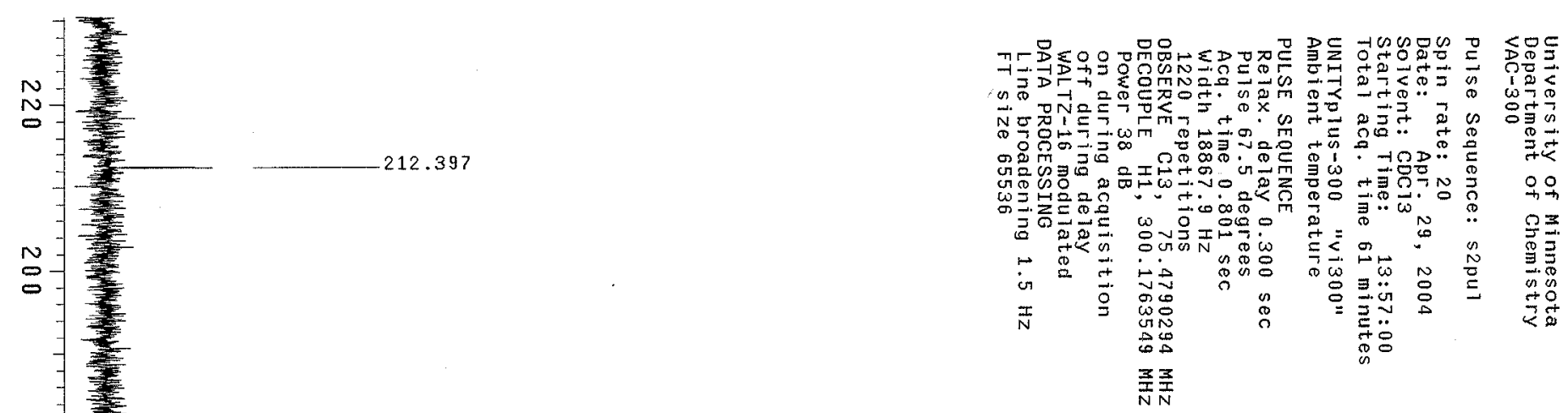

官

항

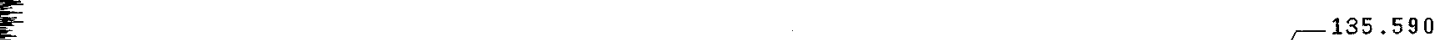

$-133.820$

129.54

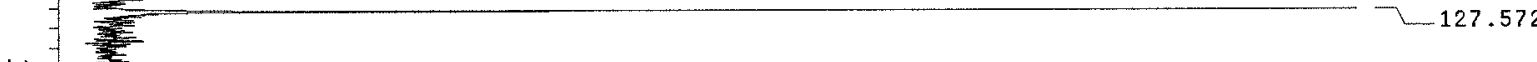

$\stackrel{\leftarrow}{\circ}$

- 106.211

$\stackrel{\circ}{\circ}$

$\stackrel{\infty}{\circ}$

$-84.637$

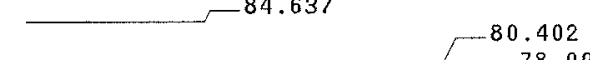

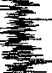

$$
\text { 。 }
$$

of

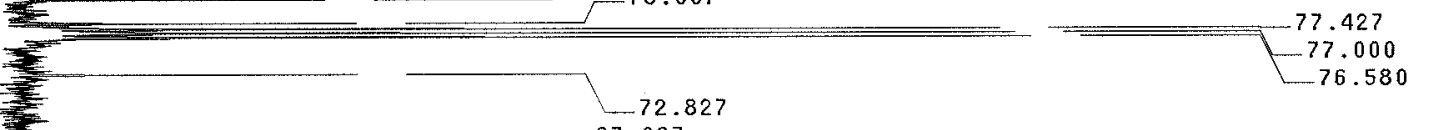

:

43.387

43.387
39.603

$-38.32$

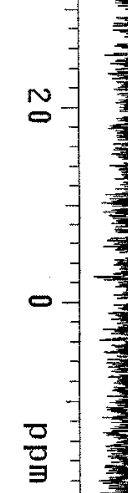

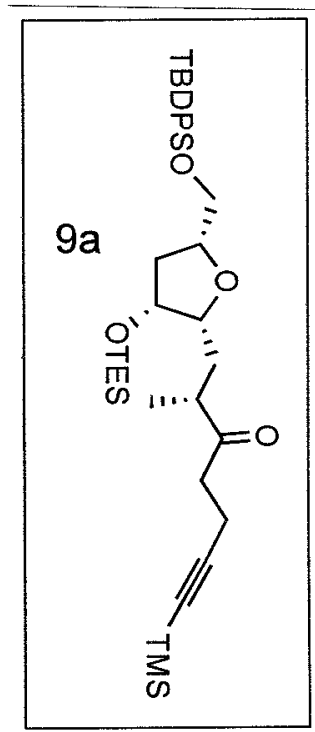



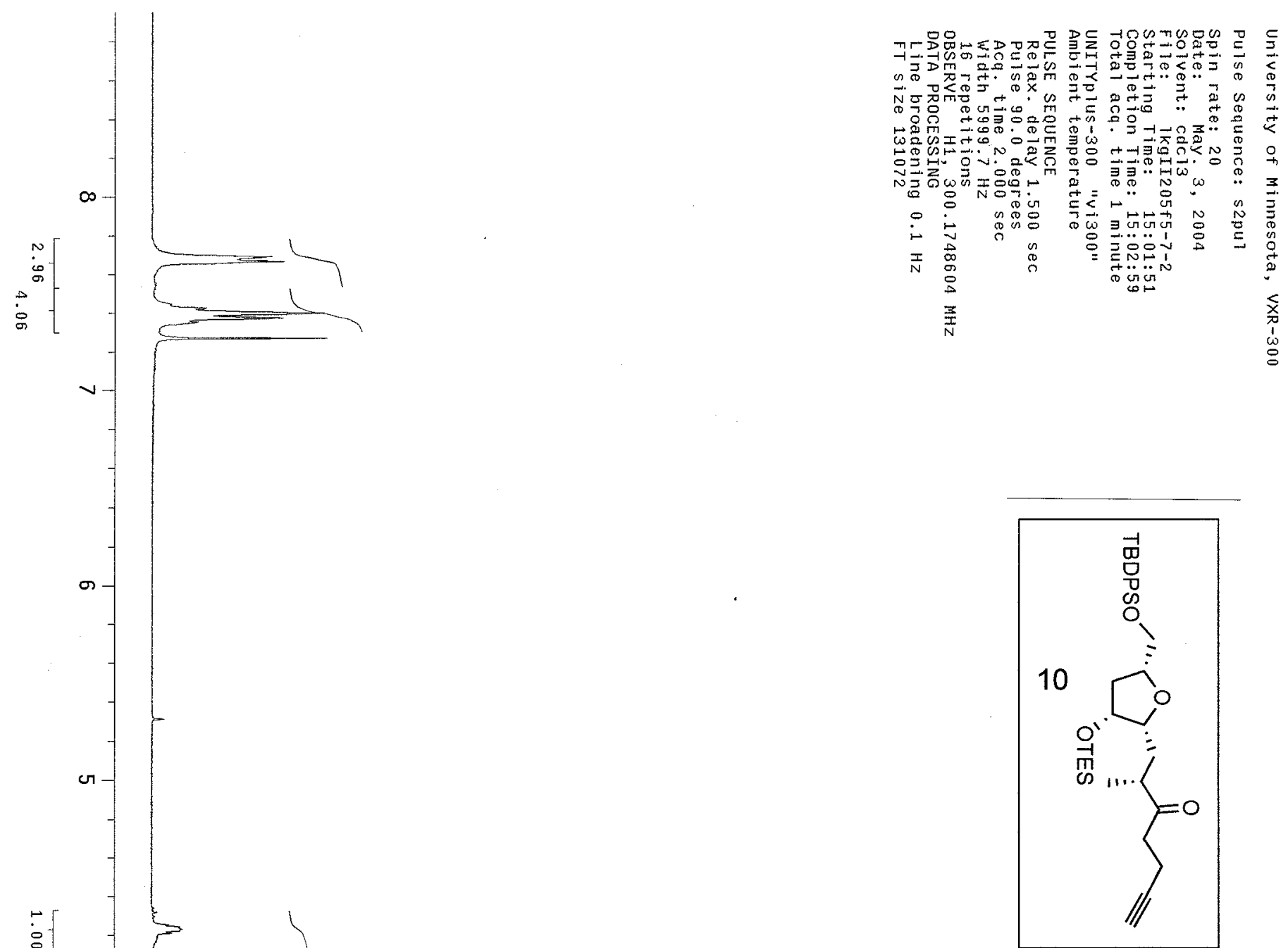


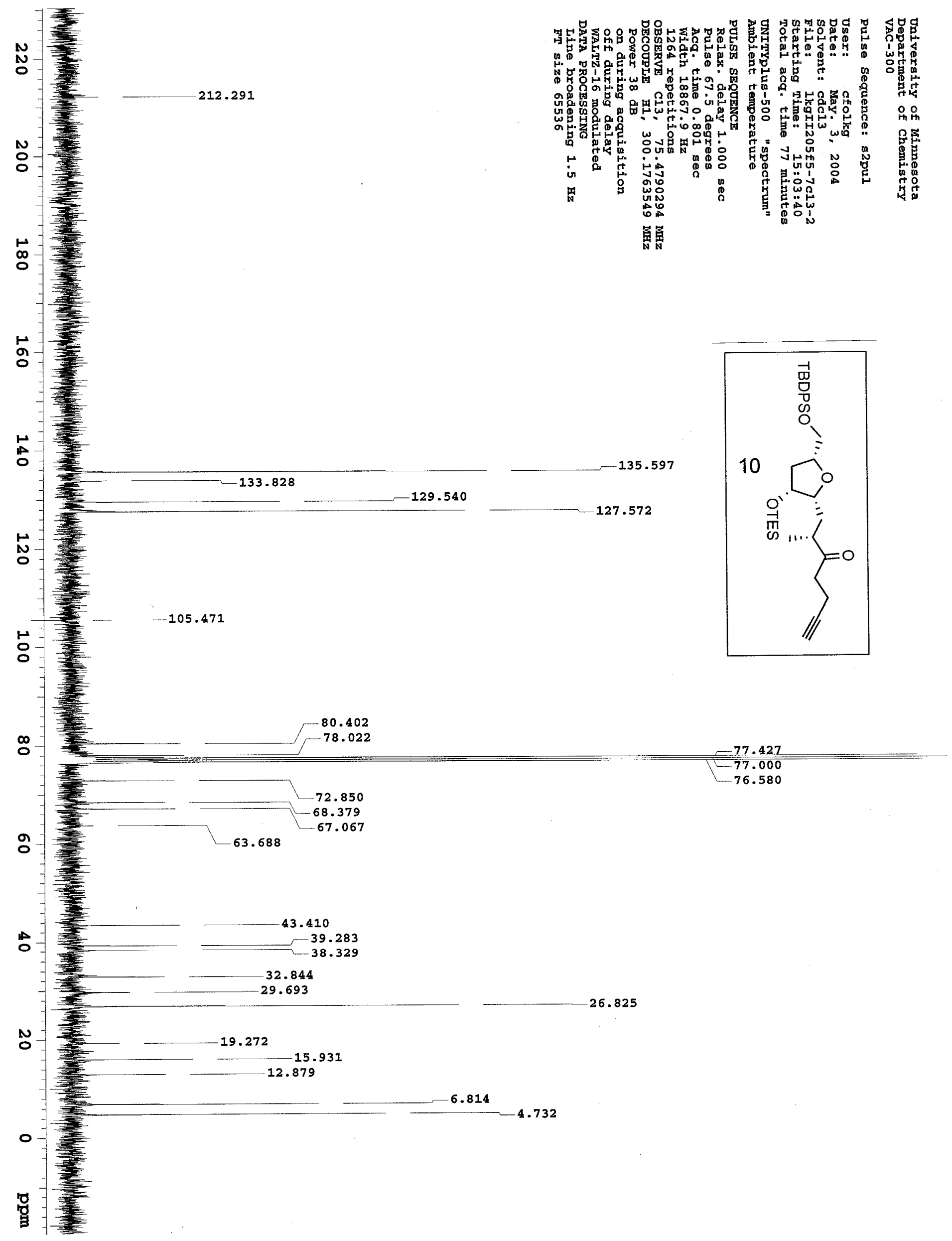




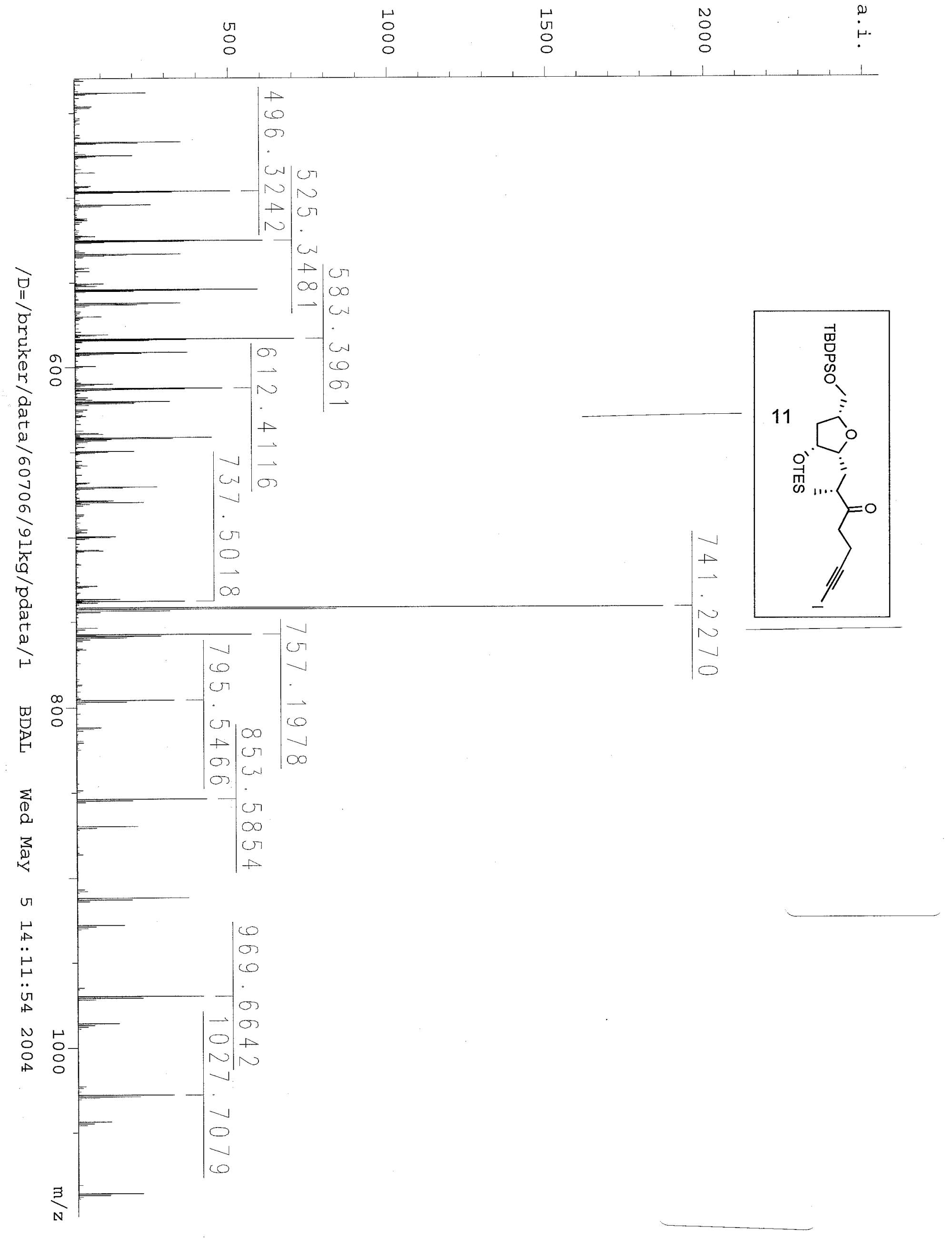




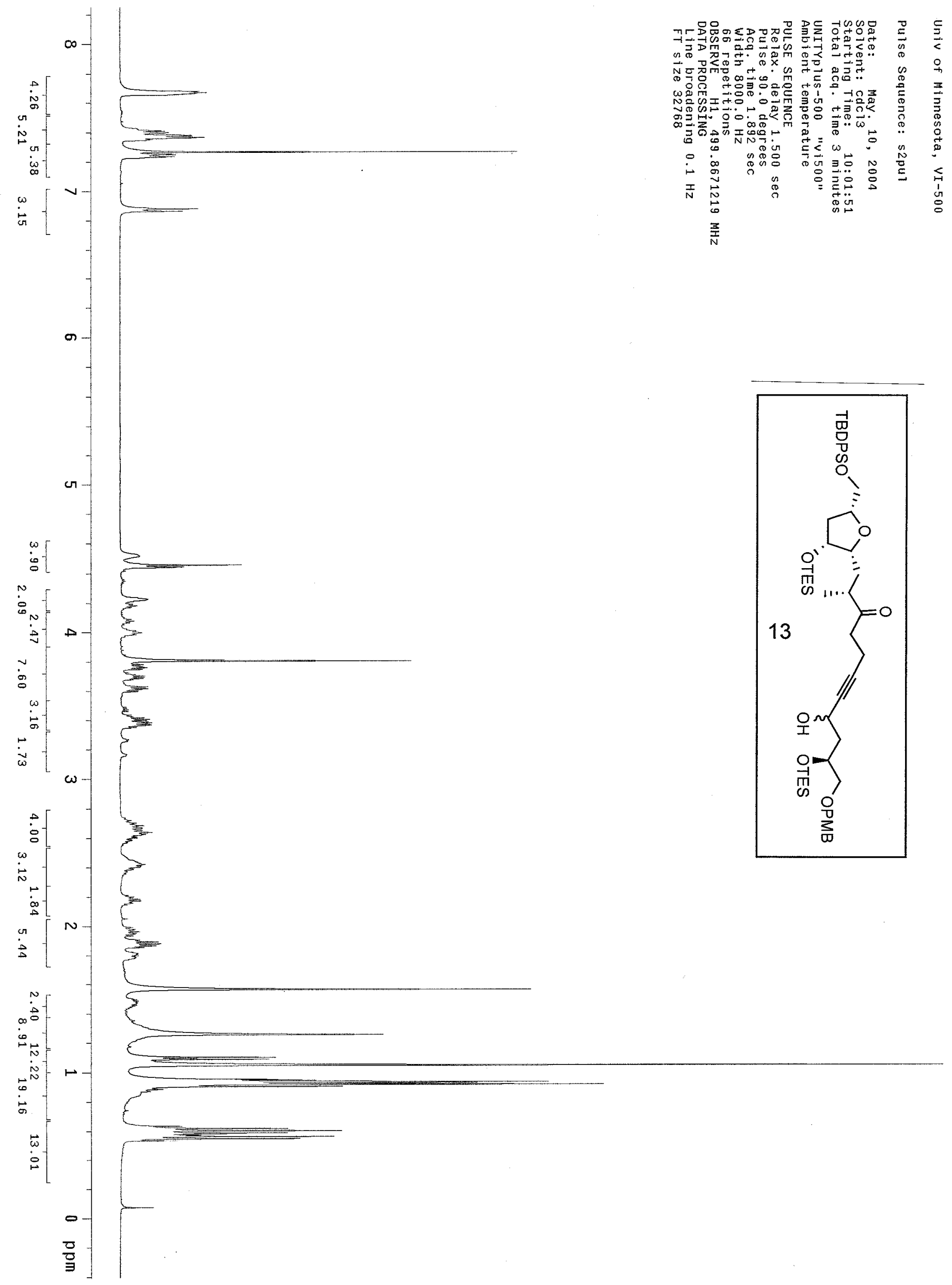



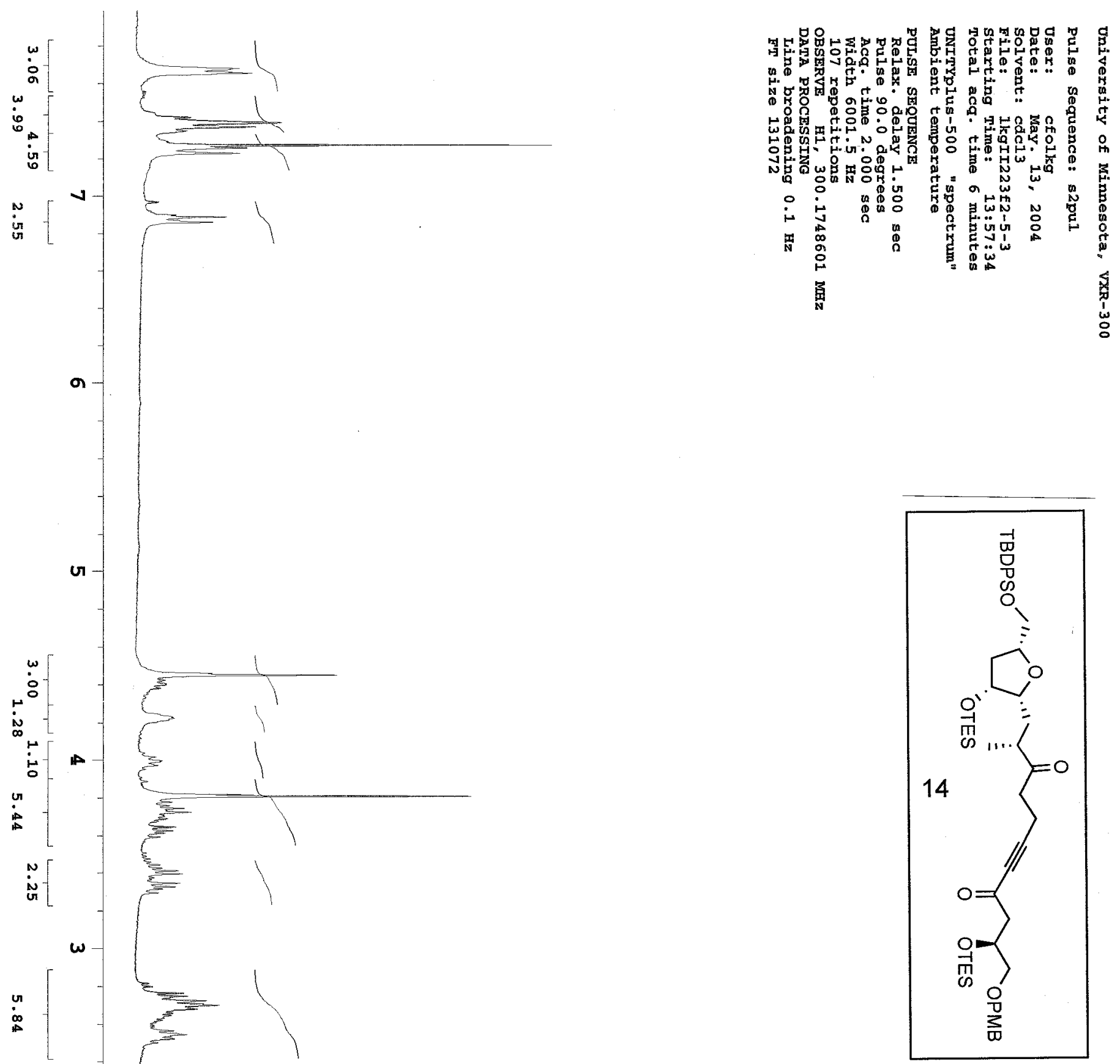


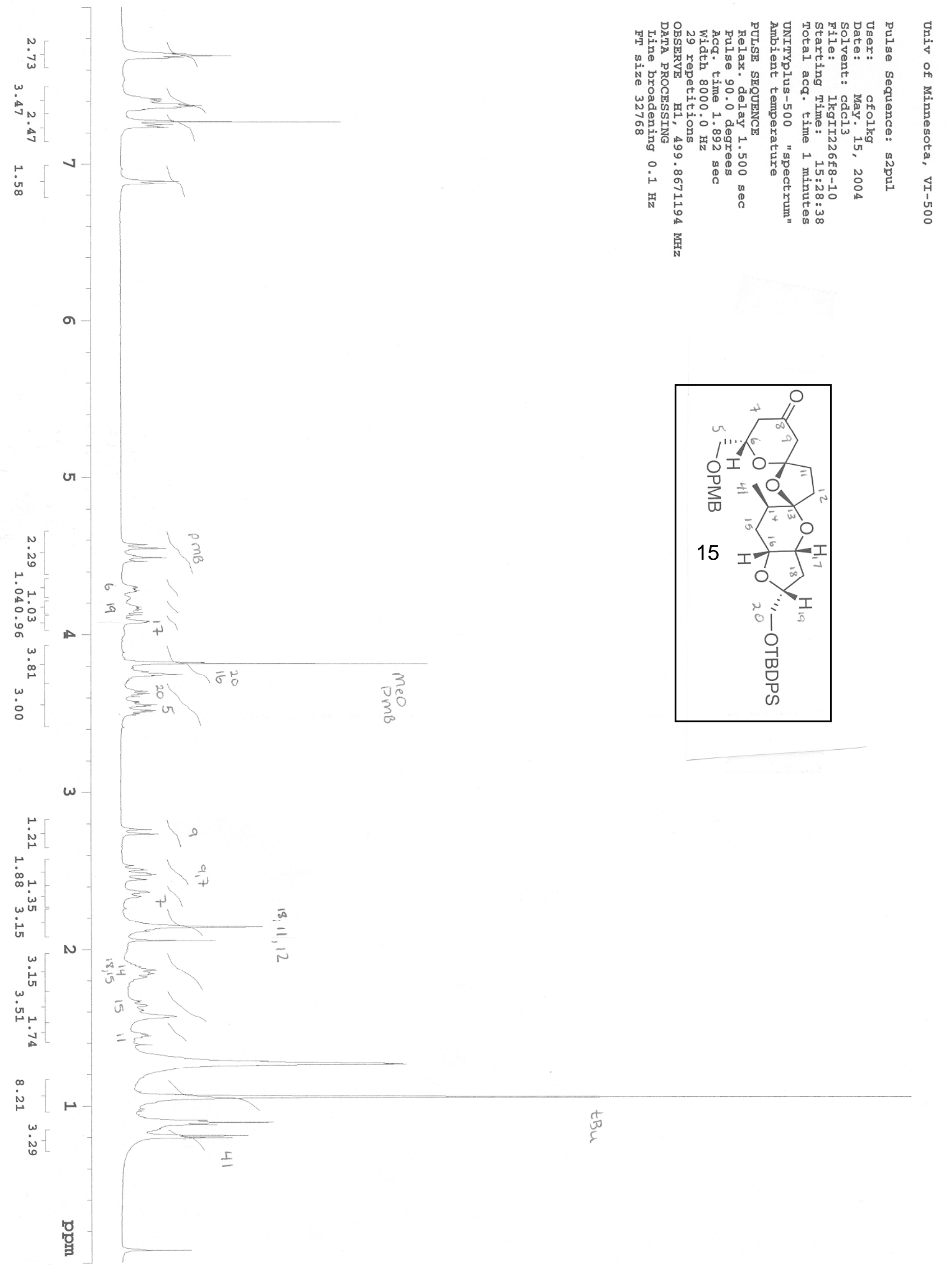




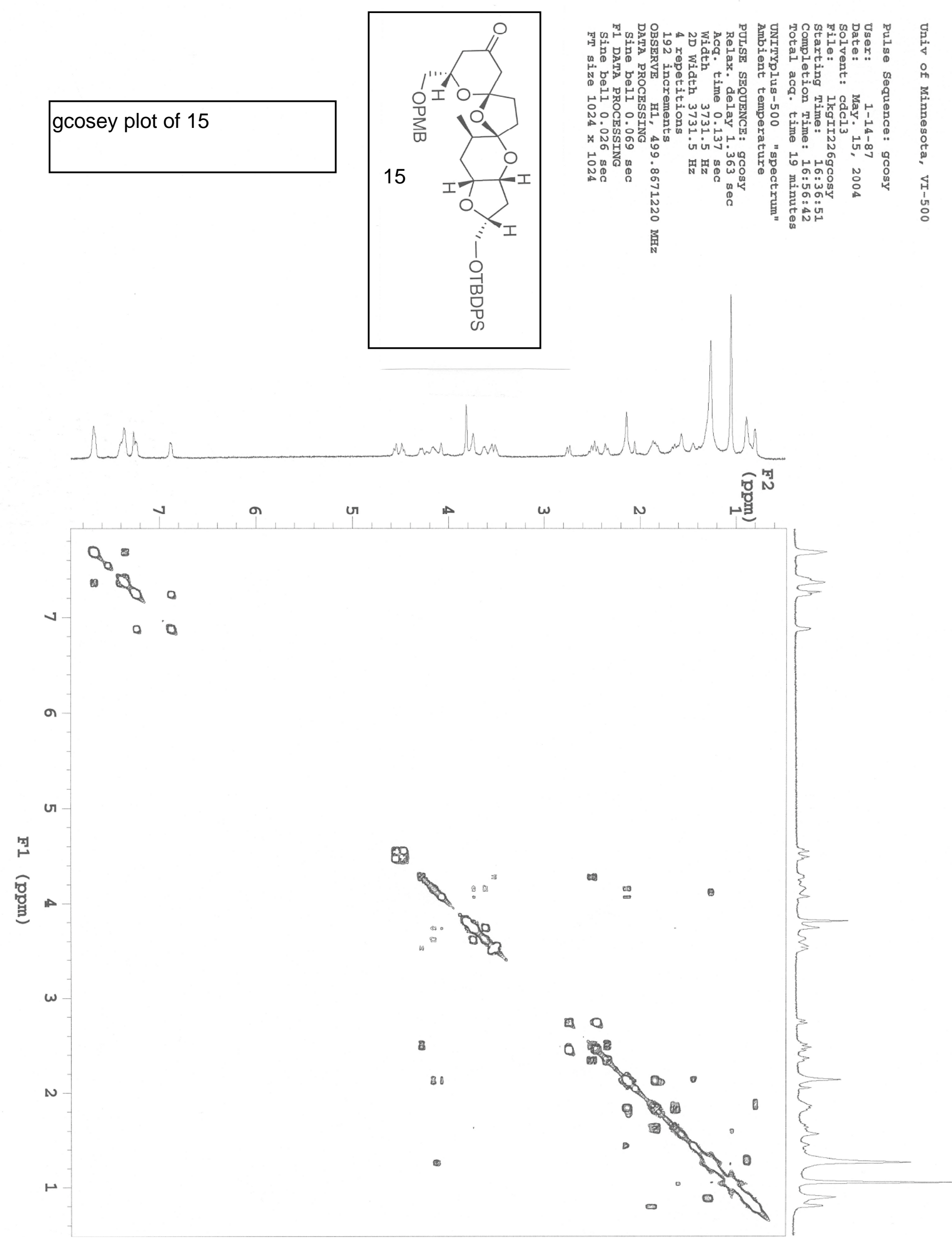



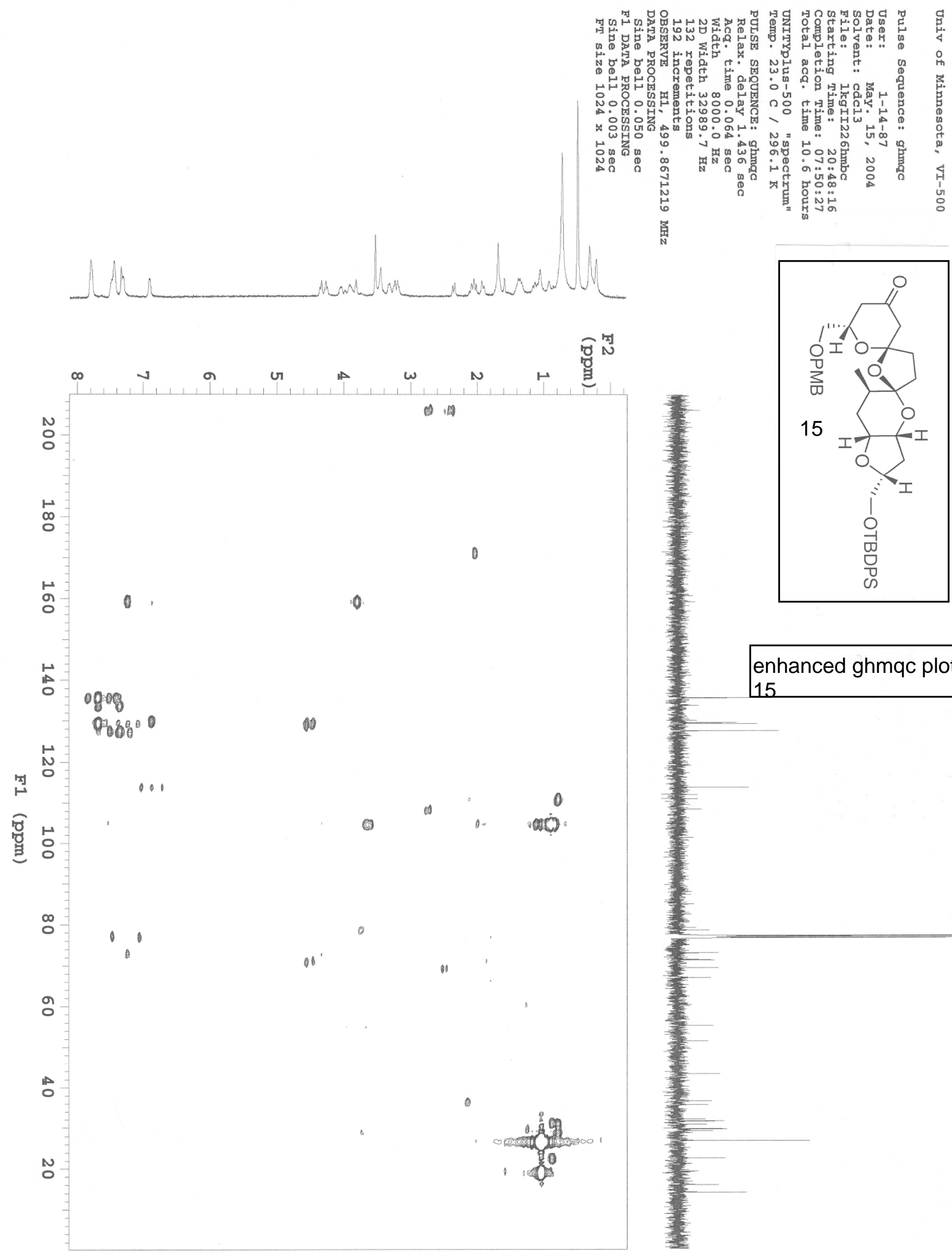

enhanced ghmqc plot of 15 


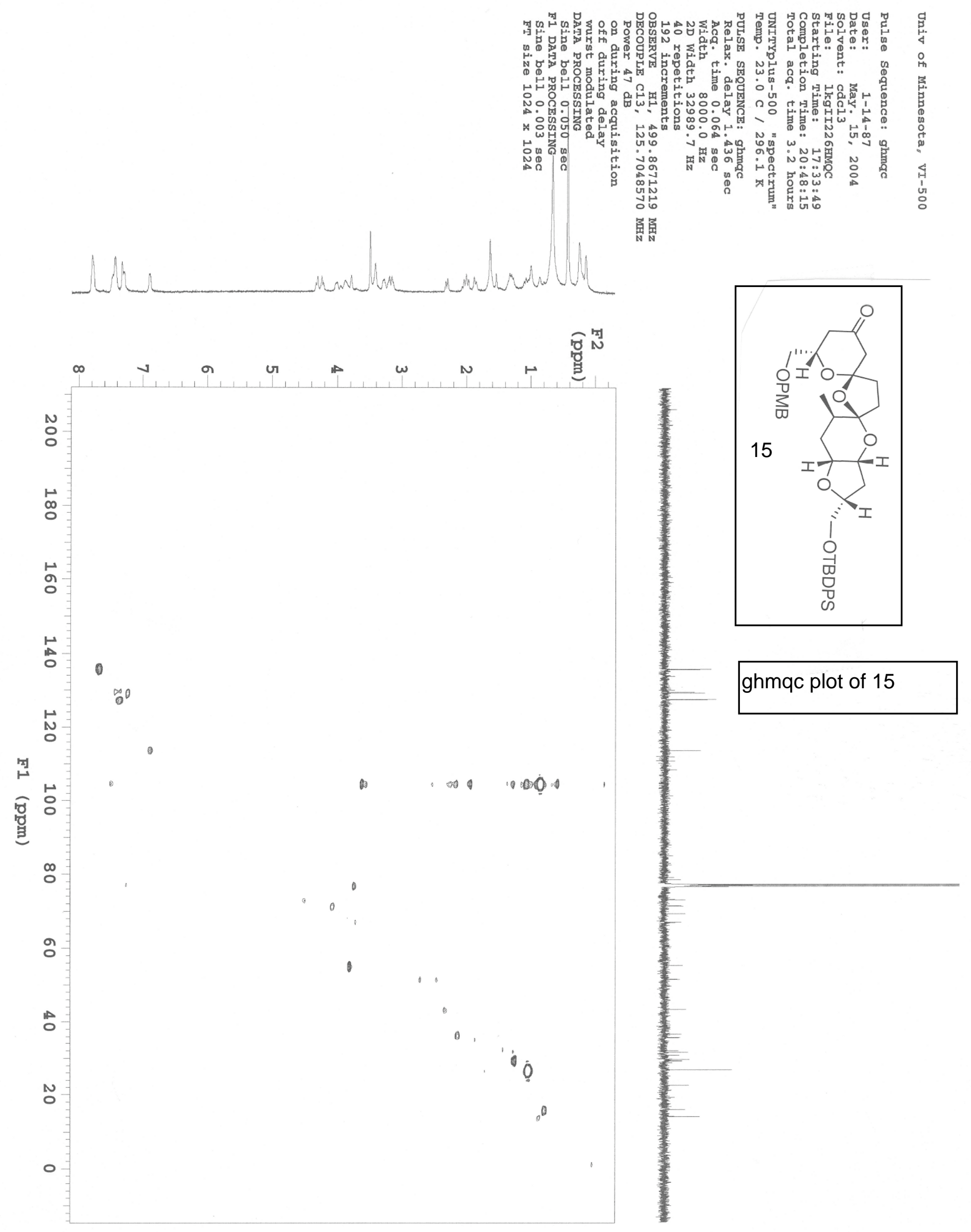



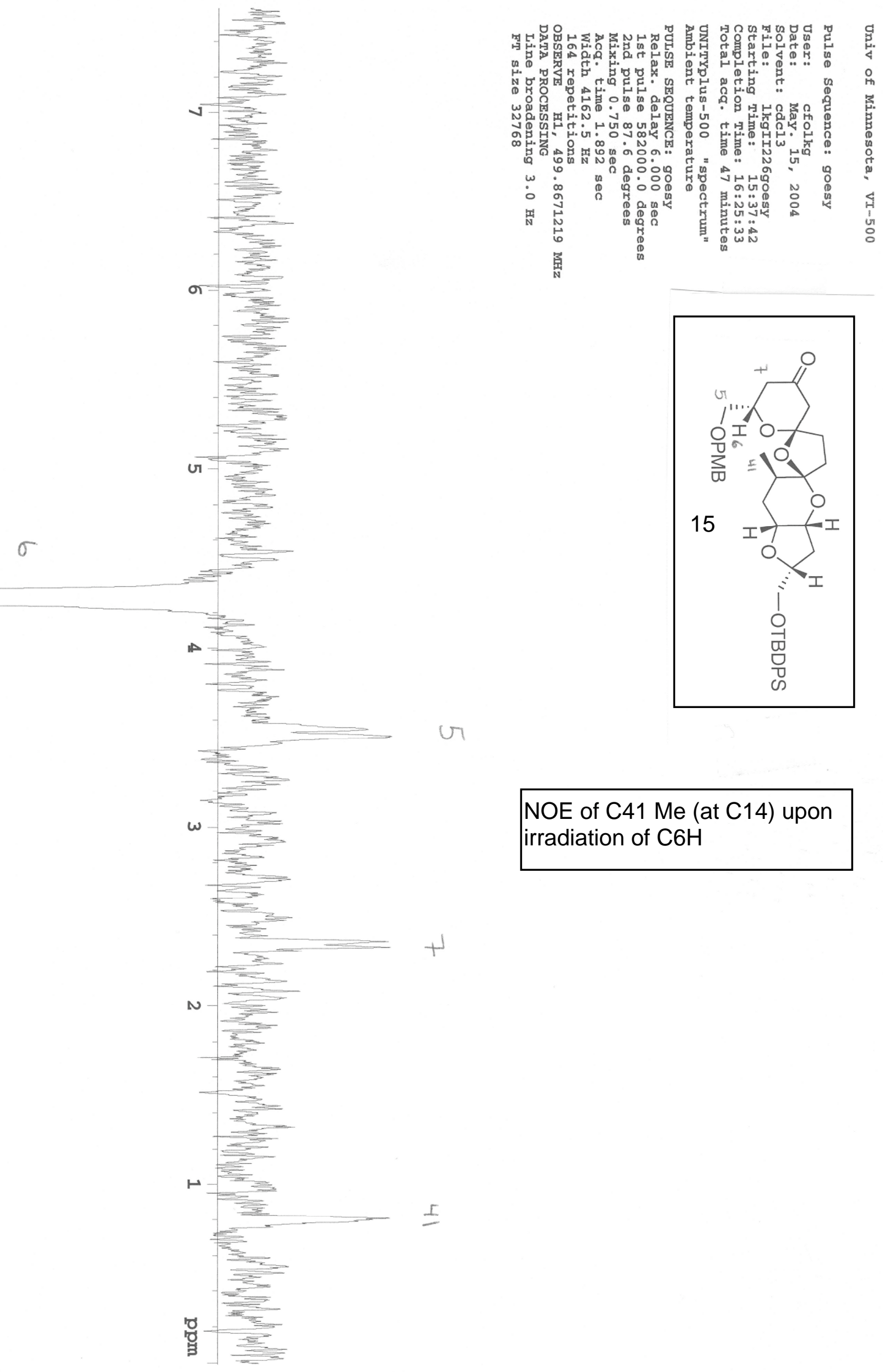

NOE of C41 Me (at C14) upon irradiation of $\mathrm{C} 6 \mathrm{H}$ 


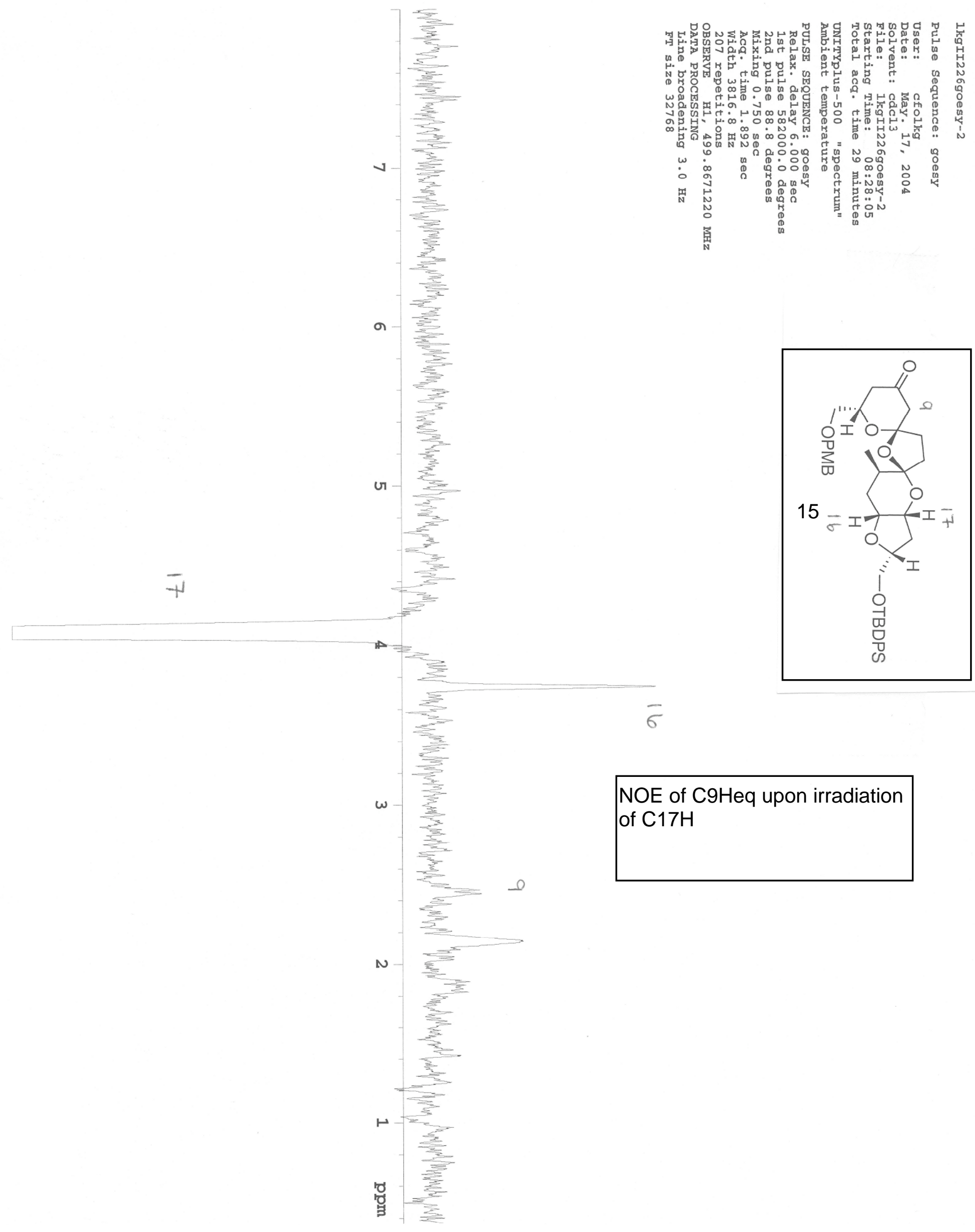




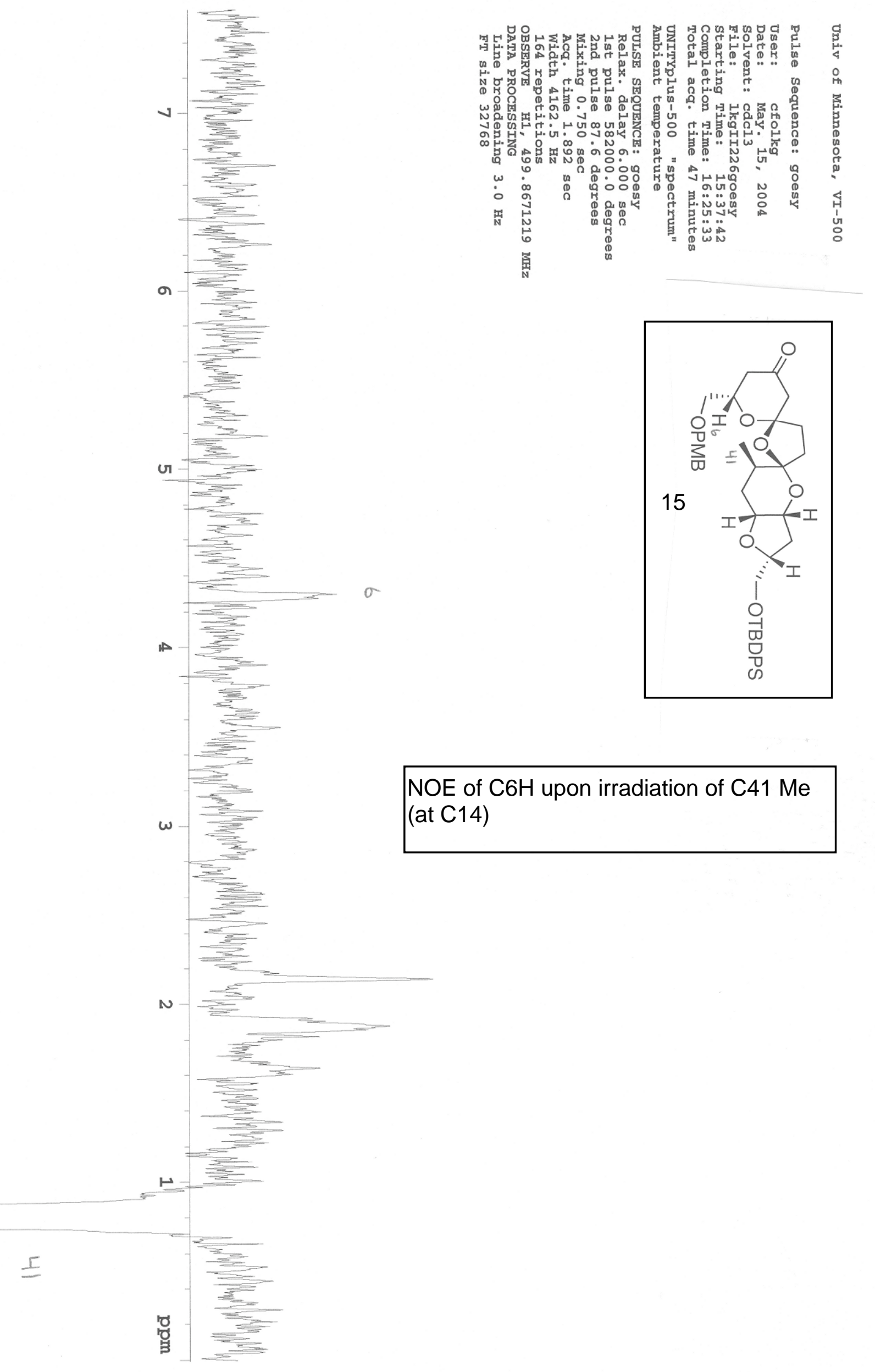

